\title{
Variability in ultra cool dwarfs: Evidence for the evolution of surface features
}

\author{
C. A. L. Bailer-Jones and R. Mundt \\ Max-Planck-Institut für Astronomie, Königstuhl 17, 69117 Heidelberg, Germany
}

Received 17 October 2000 / Accepted 30 November 2000

\begin{abstract}
We present photometric light curves for a sample of 21 ultra cool M and L dwarfs in the field and in the young open clusters $\sigma$ Orionis and the Pleiades. The list of targets includes both low mass hydrogen burning stars and brown dwarfs. Evidence for variability with rms amplitudes (in the $I$ band) of 0.01 to 0.055 magnitudes on timescales of 0.4 to 100 hours is discovered in half of these objects. Power spectral analysis using the CLEAN algorithm was performed to search for evidence of periodic variability. Some objects show strong periodicities at around a few hours, which could be due to rotational modulation of the light curve by surface features. However, several objects do not have any significant periodicities to explain their variability. The $v \sin i$ values of a similar population of objects makes it very likely that our time sampling was sensitive to the expected range of rotation periods, and simulations show that we would have detected these if they were caused by long-lived surface features. We argue that this absence of periodicity is due to the evolution of the brightness, and presumably also the physical size, of surface features on timescales of a few to a few tens of hours. This is supported in the case of 2M1145 for which two light curves have been obtained one year apart and show no common periodicity. The surface features could plausibly be photospheric dust clouds or magnetically-induced spots. The recently observed decline in chromospheric activity for late type $\mathrm{M}$ and $\mathrm{L}$ dwarfs hints towards the former explanation for at least our later-type objects. Furthermore, our sample suggests that variability is more common in objects later than M9, indicating that the variability may be related to dust formation. One light curve shows a brief, but significant, dip, which could be a short-lived feature or possibly an eclipse by a companion.
\end{abstract}

Key words. methods: data analysis - stars: atmospheres - stars: low-mass, brown dwarfs - stars: rotation stars: starspots - stars: variables: others

\section{Introduction}

Time-resolved observations are an important method for investigating astrophysical phenomena. This is particularly the case for objects which cannot be resolved spatially, as then the amount of information available is greatly limited. Temporal monitoring is central to many parts of astrophysics, such as pulsars, the physics of stars in the instability strip, microlensing and gamma ray bursts. However, monitoring is important even for apparently "stable" objects, e.g. for the determination of stellar rotation periods, and has led to the discovery of transient activity in a whole range of astrophysical objects.

Variability is a phenomenon which is potentially important in ultra cool dwarfs, because at these low temperatures (and masses) these objects are fully convective, and many molecules and condensates form in their atmospheres. Furthermore, many may also be rapid

Send offprint requests to: C. A. L. Bailer-Jones,

e-mail: calj@mpia-hd.mpg.de rotators, providing a possible driving mechanism for atmospheric dynamics. They could, therefore, show a range of time-dependent observable phenomena, such as the rotational modulation of the light curve due to surface inhomogeneities, the evolution of magnetically-induced star spots, accretion activity (for the youngest objects), flaring, movement of photospheric clouds, and eclipses by unseen companions or disks.

Ultra cool dwarfs can be divided into the three spectral types $\mathrm{T}, \mathrm{L}$ and late $\mathrm{M}$. The $\mathrm{L}$ dwarfs are the low temperature continuation of the $\mathrm{M}$ dwarf sequence. As the temperature drops, the strong $\mathrm{TiO}$ and $\mathrm{VO}$ bands which characterises the optical and infrared spectra of $\mathrm{M}$ dwarfs are replaced by very broad neutral alkali lines and lines of iron hydrides. Modelling of low resolution optical and near infrared spectra implies a temperature range of $2000 \mathrm{~K}$ down to $1300 \mathrm{~K}$ (Kirkpatrick et al. 2000). However, a temperature assignment using fits to high resolution profiles of the alkali lines indicates a somewhat hotter range of 2200-1600 K (Basri et al. 2000). At even lower 
temperatures methane can form, and broad absorption features of this - as well as water - in the infrared are the distinguishing features of $\mathrm{T}$ dwarfs. Ultra cool dwarfs cover a range of masses (the mass for a given effective temperature depending on the age) from a few Jupiter masses up to a few tenths of a solar mass $\left(1 M_{\odot}=1050 M_{\text {Jup }}\right)$. For example, an L dwarf could, in principle, be a hydrogen burning star, a brown dwarf or even a giant gas planet if it is young enough. Objects later than L4.5 are expected to be substellar (Kirkpatrick et al. 2000).

To date, little variability monitoring of ultra cool dwarfs has been reported. Tinney \& Tolley (1999) found variability (at a $98 \%$ confidence level) with an amplitude of 0.04 magnitudes over a few hours in an M9 brown dwarf, but detected no variability above 0.1 magnitudes in an L5 dwarf. Terndrup et al. (1999) searched for rotational modulation of the light curves of eight $M$ type stars and brown dwarfs in the Pleiades. They derived periodicities for two low mass stars, but found no significant variability in the rest of the sample. At the lower end of the temperature scale, Nakajima et al. (2000) found variability in the near infrared spectrum of a $\mathrm{T}$ dwarf over a period of $80 \mathrm{~min}$. In an earlier paper we reported the first results from a program to monitor a number of brown dwarfs and L dwarfs (Bailer-Jones \& Mundt 1999, hereafter Paper I). Of the six objects monitored, we discovered evidence for variability in the field L1 dwarf 2M1145, and tentatively assigned a period. In the present paper we have extended this work to a total of $21 \mathrm{M}$ and $\mathrm{L}$ dwarfs, and look for evidence of any variability in the $I$ band down to a precision of 0.005 magnitudes on timescales between a fraction of an hour and several days.

In the next section we describe the selection of the target objects and their relevant properties. Section 3 describes the observational and data reduction strategy, with a discussion of the steps required to achieve high precision relative photometry on these faint objects, as well as an accurate estimate of the photometric errors. We then discuss the construction and analysis of the differential light curves to look for evidence of variability. Section 4 describes our time series analysis techniques. The results section summarizes our findings, with a description for individual objects. The main argument of this paper is presented in Sect. 6, where we discuss the interpretation of our results in terms of physical phenomena. The data presented in Paper I have been re-reduced and re-analysed in the present paper. Although the results are generally consistent, the results in the present paper supersede those in Paper I.

\section{Target selection}

Our sample consists of both L dwarfs and late M dwarfs. The targets were chosen on the basis of being (a) observable for a large fraction of the night in one observing run, and (b) sufficiently bright that a good SNR (signalto-noise ratio) could be achieved in a short integration time (see Sect. 3.1). Within these selection constraints, we then attempted to observe objects with a range of spectral types. Details of the 21 observed objects are given in Table 1. Ten are field L dwarfs. At the time of the observations, essentially the only available L dwarfs were the 25 listed by Kirkpatrick et al. (1999) (most of which were discovered by 2MASS, the Two Micron All Sky Survey), plus (for the most recent observating run only) a handful from the Sloan Digital Sky Survey (SDSS). The ages ${ }^{1}$ of these objects are generally unknown, but are probably of order 1 Gyr. The other 11 objects in Table 1 are cluster objects. Five are members of the Pleiades (age $120 \mathrm{Myr}$ ), of which two (Teide 1 and Calar 3) are confirmed brown dwarfs, two (Roque 11 and Roque 12) are probably brown dwarfs, and the last (Roque 16) is very close to the hydrogen burning limit so its status is uncertain. The six remaining objects are candidate members of the $\sigma$ Orionis cluster, with masses between 0.02 and $0.12 M_{\odot}$, part of this range reflecting the uncertainty in the cluster age of 1-5 Myr. The four faintest objects in this last cluster were observed because they just happened to be in the field of another target.

\section{Data aquisition and reduction}

\subsection{Observations}

The data were obtained over three observing periods: January 1999 (AJD 1187.4-1192.8, hereafter 99-01), September 1999 (AJD 1432.8-1436.2, hereafter 99-09) and February 2000 (AJD 1601.8-1607.2, hereafter 00-02). AJD is an adjusted Julian day ${ }^{2}$, equal to the Julian Day minus 2450000. In all cases the CAFOS instrument on the $2.2 \mathrm{~m}$ telescope at the Calar Alto Observatory (Spain) was used. The objects were observed in the $I$ filter because of their very red optical colours. The $99-01$ run used a $1 \mathrm{~K} \times 1 \mathrm{~K}$ TEK CCD with a $9^{\prime} \times 9^{\prime}$ field of view; the other two runs used a SITe $2 \mathrm{~K} \times 2 \mathrm{~K}$ CCD windowed to a field of view of $9^{\prime} \times 11^{\prime}$ (to reduce the readout time). In all cases the pixel scale was $0.53^{\prime \prime} /$ pix.

To ensure a good variability detection efficiency, we decided that the magnitude error in the target star should be no more than 0.01 magnitudes at each epoch (i.e. SNR > 110). On the one hand, a long integration time is required to achieve this high SNR, but on the other hand a short one is required to ensure we do not "blur out" the variable phenomenon we are trying to observe. A simple calculation shows that when observing a sinusoidal variation of period $\tau$ with an integration time of $t$, then a maximum error of $3.1 t / \tau$ (or typical error of $2.2 t / \tau$ ) in units of the peak-to-peak amplitude is introduced (provided $\sin (\pi t / \tau) \simeq \pi t / \tau)$. Tolerating a maximum blurring error of 0.2 , and assuming that no period of interest is

\footnotetext{
${ }^{1}$ Gizis et al. (2000) investigate the dating of $\mathrm{L}$ dwarfs based on activity and kinematics.

${ }^{2}$ In Paper I, the observing dates were incorrectly referred to as MJD instead of JD.
} 
Table 1. Properties of ultra cool dwarf targets. Each reference makes use of a different $I$ band and even definition of magnitude, so values are only intended to be indicative. In particular, the SDSS $I$ filter is somewhat bluer than the Cousins $I$ filter, thus yielding fainter magnitudes for $\mathrm{L}$ dwarfs. The spectral types in parentheses have been estimated from the $R-I$ colours of Béjar et al. (1999)

\begin{tabular}{|c|c|c|c|c|c|c|}
\hline name & IAU name & $I$ & SpT & $\begin{array}{l}\mathrm{H} \alpha \mathrm{EW} \\
\AA\end{array}$ & $\begin{array}{l}\text { Li I } \lambda 6708 \mathrm{EW} \\
\AA\end{array}$ & reference \\
\hline $2 \mathrm{M} 0030$ & 2MASSW J0030438+313932 & 18.82 & L2 & $4.4 \pm 0.2$ & $<1.0$ & Kirkpatrick et al. (1999) \\
\hline $2 \mathrm{M} 0326$ & 2MASSW J0326137+295015 & 19.17 & L3.5 & $9.1 \pm 0.2$ & $<1.0$ & Kirkpatrick et al. (1999) \\
\hline $2 \mathrm{M} 0345$ & 2MASSW J0345432+254023 & 16.98 & L0 & $\leq 0.3$ & $<0.5$ & Kirkpatrick et al. (1999) \\
\hline 2M0913 & 2MASSW J0913032+184150 & 19.07 & L3 & $<0.8$ & $<1.0$ & Kirkpatrick et al. (1999) \\
\hline 2M1145 & 2MASSW J1145572+231730 & 18.62 & $\mathrm{~L} 1.5$ & $4.2 \pm 0.2$ & $<0.4$ & Kirkpatrick et al. (1999) \\
\hline 2M1146 & 2MASSW J1146345+223053 & 17.62 & L3 & $\leq 0.3$ & $5.1 \pm 0.2$ & Kirkpatrick et al. (1999) \\
\hline 2M1334 & 2MASSW J1334062+194034 & 18.76 & L1.5 & $4.2 \pm 0.2$ & $<1.5$ & Kirkpatrick et al. (1999) \\
\hline 2M1439 & 2MASSW J1439284+192915 & 16.02 & L1 & $1.13 \pm 0.05$ & $<0.05$ & Reid et al. (2000) \\
\hline SDSS 0539 & SDSSp J053951.99-005902.0 & 19.04 & L5 & & & Fan et al. (2000) \\
\hline SDSS 1203 & SDSSp J120358.19+001550.3 & 18.88 & L3 & & & Fan et al. (2000) \\
\hline Calar 3 & & 18.73 & M9 & $6.5-10.2$ & $1.8 \pm 0.4$ & Rebolo et al. (1996) \\
\hline Roque 11 & RPL J034712+2428.5 & 18.75 & M8 & $5.8 \pm 1.0$ & & Zapatero Osorio et al. (1999) \\
\hline Roque 12 & & 18.47 & M7.5 & $19.7 \pm 0.3$ & $\leq 1.5$ & Martín et al. (1998) \\
\hline Roque 16 & RPL J034739+2436.4 & 17.79 & M6 & $5.0 \pm 1.0$ & & Zapatero Osorio et al. (1999) \\
\hline Teide 1 & TPL J034718+2422.5 & 18.80 & M8 & $3.5-8.6$ & $1.0 \pm 0.2$ & Rebolo et al. (1995) \\
\hline S Ori 31 & S Ori J053820.8-024613 & 17.31 & $(\mathrm{M} 6.5)$ & & & Béjar et al. (1999) \\
\hline S Ori 33 & S Ori J053657.9-023522 & 17.38 & $(\mathrm{M} 6.5)$ & & & Béjar et al. (1999) \\
\hline S Ori 34 & S Ori J053707.1-023246 & 17.46 & $(\mathrm{M} 6)$ & $\leq 5.0$ & & Béjar et al. (1999) \\
\hline S Ori 44 & S Ori J053807.0-024321 & 19.39 & M6.5 & $60.0 \pm 1.0$ & & Béjar et al. (1999) \\
\hline S Ori 45 & S Ori J053825.5-024836 & 19.59 & M8.5 & & & Béjar et al. (1999) \\
\hline S Ori 46 & S Ori J053651.7-023254 & 19.82 & $(\mathrm{M} 8.5)$ & & & Béjar et al. (1999) \\
\hline
\end{tabular}

below 1-2 hours, we arrive at a maximum ${ }^{3}$ integration time of around 4-8 min. A constant integration time of five minutes was used during 99-01, later increased to eight minutes for the subsequent two runs. The only exception was the brighter target 2M1439, for which an integration time of $80 \mathrm{~s}$ was used to avoid saturation. The eight minute integration time then set the faintest magnitude limit of the targets at around $I=19.0$. Within each night, objects were observed in a repetitive cycle, although not all objects were observed every night. During 00-02, two images of the same target were often taken in each cycle.

\section{2. $C C D$ processing}

The data from 99-01 were presented in Paper I, but have been re-reduced for the present paper in exactly the same manner as the other two runs. The reduction procedure is now described.

\subsubsection{Basic reduction}

A one-dimensional bias was subtracted from each frame using the overscan region in each frame. A small residual two-dimensional bias pattern remained, and this was removed by subtracting a low-order fit to a median-combination of many zero-length dark exposures.

\footnotetext{
${ }^{3}$ Such an integration time does not automatically preclude detection of shorter periods, because multiple observations are made at different parts of any sinusoidal curve, but the sensitivity to very short periods may be reduced.
}

The variable sensitivity across the detector was corrected using illumination-corrected dome flats, in the following way. Several dome flats taken through the same optical path (i.e. no telescope or lamp movemement) were averaged with outlier clipping. While this is sufficient to remove the small scale pixel-to-pixel variations, it will not correct the large scale variations, on account of the different illuminations from the dome wall and the night sky. Thus the global illumination of this combined dome flat was removed (by dividing it by its own low-order fit) and replaced with the global illumination of the sky. This global sky illumination was obtained by making a loworder fit to a median-combination of a large number (typically 40-50) of night sky images of different fields. (These frames were selected from the science frames plus a number of images of dark patches of sky using the same integration time.) These emphasised points are necessary to ensure that bright stars are removed and do not distort the fit. The resulting corrected dome flat is normalised to have unit mean, and each science frame frame divided by it. This procedure was done separately for each night of each run.

\subsubsection{Fringe removal}

Most frames showed interference fringes caused by narrow line emission from the Earth's atmosphere interfering in the non-uniformly-thick layer on the CCD. The flux amplitude was typically $2 \%$ of the sky level (a few times the sky noise), and the spatial scale of order a hundred 
pixels, so it was essential that these fringes be removed. Within a given observing run the fringe pattern was found to be stable, i.e. independent of time or telescope point$\mathrm{ing}^{4}$. It is important to realise that fringing is an additive phenomenon. Thus the fringes must be subtracted from the science frames; they must not be divided, e.g. using the flat field, as they have not modulated the star light. Similarly, the flat field itself must not have fringes, and it was for this reason that twilight flats could not generally be used. The fringes were removed by constructing a "fringe correction" frame, which is a median combination of a set of flat-fielded night sky images (the same set as used for creating the sky illumination). Taking the median at each pixel is necessary to remove the stars, but this works only if all the frames have a common flux zero point with respect to these stars, i.e. are sky-subtracted: due to varying airmass or the presence of thin cloud and the Moon, the sky level often differed. Thus before combination, a low-order fit to each frame was subtracted. The resulting fringe correction frame showed only the fringes, but was fairly noisy. This was improved with a spatial smoothing (a boxcar filter of size three pixels). To first order, the scale of the fringes in a frame is proportional to the exposure time, so subtracting the smoothed fringe frame usually removed the fringes. However, in some cases this over- or under-subtracted the fringes, presumably because the strength of the fringe pattern also depends on both the airmass and degree of (thin) cloud cover. In these cases a factor of the smoothed fringe frame was subtracted, the factor (in the range 0.3 to 2.8 ) determined manually.

\subsubsection{Error sources}

As will be seen in Sect. 3.3, an accurate knowledge of the photometric errors (or at least, not an underestimate) is required for the detection of variability. For our brighter objects, the quality of the flat field and the fringe removal set a limit to the photometric precision. Through various tests we determined that these contribute random errors in the photometry of no more than $0.5 \%$. Other effects which are significantly less could be ignored (see Paper I). Non-linearity in the response of the detector was checked and could be ignored for flux levels of interest. A spatial non-linearity due to the shutter was avoided by using sufficiently long integration times $(>20 \mathrm{~s})$ in all frames. The CAFOS instrument suffers from geometric distortion, specifically a change in the pixel scale with distance from the optical axis. As the different images of a target field were not always identically positioned with respect to this axis, this potentially introduces errors into relative photometry. While it can be corrected for, it was found that it contributed an error in the relative photometry of no more than $0.1 \%$.

\footnotetext{
4 The telescope re-pointing accuracy was often good only to tens of pixels, meaning that in a sequence of frames the image of a given field moved relative to the fringe pattern.
}

\subsection{Photometry}

To reduce sensitivity to temporal variations in the Earth's atmosphere through which the target must be observed, the flux of the target is monitored relative to a number of reference stars in the field. These were chosen according to the following criteria:

1. near-Gaussian, near-circular point spread function (PSF), i.e. not an extended object;

2. isolated from other sources;

3. present on every frame;

4. bright (generally brighter than the target, although in several cases the target was one of the brighter nonsaturated stars). It was also ensured that the flux was less than about $75 \%$ of the saturation of the analogueto-digital convertor (to avoid non-linearity).

Aperture photometry was performed on the target and reference stars in each frame. The choice of aperture size was discussed in Paper I. Although the photometry (and light curve analysis) was done in a range of aperture sizes, results are presented using an aperture radius of 3.5 pixels, which maximises the SNR while reducing all systematic errors below the 0.5\% level discussed in Sect. 3.2.3.

A differential light curve for the target was calculated as follows. Let $F_{i}$ be the flux (in collected electrons) in the $i$ th reference star of $N$ in a frame. The reference flux in that frame is defined as

$F_{\mathrm{r}}=\frac{1}{N} \sum_{i}^{N} F_{i}$

and the reference magnitude is

$m_{\mathrm{r}}=-2.5 \log _{10} F_{\mathrm{r}}$.

The relative magnitude of the target is then defined as

$m_{\mathrm{d}}=m_{\mathrm{s}}-m_{\mathrm{r}}=2.5 \log _{10}\left(\frac{F_{\mathrm{r}}}{F_{\mathrm{s}}}\right)$

where $F_{\mathrm{s}}$ and $m_{\mathrm{s}}$ are the flux and magnitude of the target respectively. We chose to form $m_{\mathrm{r}}$ by averaging fluxes rather than magnitudes, as this gives more weight to the brighter, higher SNR objects: Simply averaging magnitudes gives almost as much weight to the faintest reference stars $(I \sim 19)$ as to the brightest $(I \sim 16)$. More "sophisticated" weighting schemes did not create a more precise reference light curve. If $m_{\mathrm{d}}(k)$ is the relative magnitude in frame $k$, then the light curve is $m_{\mathrm{d}}(1), m_{\mathrm{d}}(2), \ldots, m_{\mathrm{d}}(k), \ldots, m_{\mathrm{d}}(K)$, from which the mean is subtracted so that $\sum_{k} m_{\mathrm{d}}(k)=0$.

We assume that changes in atmospheric transparency equally affect all $\operatorname{stars}^{5}$, so if $m_{\mathrm{r}}$ is defined using nonvariable reference stars (see Sect. 4.1), changes in $m_{\mathrm{d}}$ are either due to noise or to intrinsic changes in the luminosity

\footnotetext{
5 This is reasonable if the integration time is long enough for any thin clouds to move across the whole field of view. See also Sect. 5.1
} 
of the target. To distinguish between these it is important to know the errors in $m_{\mathrm{d}}$ as accurately as possible. It can be shown that the expected error, $\delta m_{\mathrm{d}}$, in $m_{\mathrm{d}}$ is given by

$$
\left(\delta m_{\mathrm{d}}\right)^{2}=\left(\delta m_{\mathrm{s}}\right)^{2}+\left(\frac{1}{N F_{\mathrm{r}}}\right)^{2} \sum_{i}^{N} F_{i}^{2}\left(\delta m_{i}\right)^{2}
$$

where $\delta m_{\mathrm{s}}$ and $\delta m_{i}$ are the magnitude errors in the target and $i$ th reference star respectively. The error contributions were discussed in Paper I, but include: noise in the object (assumed to be Poissonian); noise in the sky (measured from the standard deviation in the sky aperture); uncertainty in the subtracted sky level; a contribution of $0.5 \%$ from the "informal" errors. This last error (dominated by imperfect flat fielding and fringe removal) may be a conservatively large estimate, but ensures increased caution in claiming to have detected variability.

\section{Time series analysis}

\section{1. $\chi^{2}$ test}

A general test of variability can be made using a $\chi^{2}$ test, in which we evaluate the probability that the deviations in the light curve are consistent with the photometric errors. The null hypothesis for this test is that there is no variability $^{6}$. We evaluate

$$
\chi^{2}=\sum_{k}^{K}\left(\frac{m_{\mathrm{d}}(k)}{\delta m_{\mathrm{d}}(k)}\right)^{2}
$$

and determine the probability, $p$, (from tables) that the null hypothesis is true. A large $\chi^{2}$ indicates greater deviation compared to the errors, and thus a smaller $p$. We claim evidence for variability if $p<0.01$. This test was first used to remove any variable reference stars, by forming the light curve of each reference star relative to all the others (see Paper I). The test was then applied to the target star using the resulting non-variable reference stars.

\subsection{Power spectrum estimation with CLEAN}

Evidence for periodic variability was then searched for using the power spectrum or periodogram. In particular, a dominant periodicity may be present at the rotation period due to rotational modulation of the light curve by surface inhomogeneities. For a continuous light curve $g(t)$, the power at frequency $\nu$ is $|G(\nu)|^{2}$, where

$G(\nu)=F T[g]=\int_{-\infty}^{+\infty} g(t) \mathrm{e}^{-2 \pi i \nu t} \mathrm{~d} t$

and $F T[g]$ denotes the Fourier transform of $g(t)$. In Paper I, this was estimated using the Lomb-Scargle periodogram, partly on the basis of the existence of a convenient significance test. However, one of the drawbacks

\footnotetext{
${ }^{6}$ As the mean has been subtracted from the light curve, the degrees of freedom for the test is $K-1$, where $K$ is the number of points in the light curve.
}

of this method is that it makes no attempt to remove the spectral window function from the data. Suppose we observe $g(t)$ at certain epochs $t_{1}, t_{2}, \ldots, t_{n}$, specified by the (discrete) sampling function $s(t)$. The observed data are then given by the (discrete) function $d(t)=g(t) s(t)$ (which in our case is just the set $m_{\mathrm{d}}(k)$ ). The power spectrum we observe is $|D(\nu)|^{2}$, given by

$D(\nu)=F T[d(t)]=G(\nu) \otimes W(\nu)$

where $W(\nu)=F T[s(t)]$ is the spectral window function and $\otimes$ is the convolution operator. Hence we do not observe the power spectrum of the process we are observing, but rather the power spectrum of its convolution with the window function. This can have serious consequences, as peaks in the power spectrum may be due to the way in which the data were sampled, and not intrinsic to the observed process itself (see, for example, Deeming 1975; Roberts et al. 1987). For equal spaced sampling, this manifests itself as aliasing. For other samplings, $W(\nu)$ can have considerable "power" at a range of frequencies, and for low SNR data can lead to periodicities in $G(\nu)$ being completely obscured in $D(\nu)$. One approach to mitigating the effects of the window function is a deconvolution of $D(\nu)$. Although a direct deconvolution is not possible, Roberts et al. (1987) modified the CLEAN algorithm (used to reconstruct two-dimensional images from interferometric data) to iteratively remove the spectral window function from the raw, or dirty, power spectrum. This works by identifying peaks in the power spectrum and subtracting the power due to the convolution of $W(\nu)$ associated with them. The resulting cleaned power spectrum, $P(\nu)$, generally consists of peaks at a number of distinct frequencies, plus a residual spectrum consisting of the noise and any spectral features not well represented by the cleaned frequency components.

We have used a CLEAN algorithm written by Harry Lehto (2000, private communication). The cleaned power spectrum is a frequency domain representation of the light curve, $g(t)$, using sinusoids of amplitude $A$ (not peak-topeak), frequency $\nu$ and phase $\phi$, determined by CLEAN. The power, $P$, at a certain frequency is related to the amplitude by $A=2 \sqrt{P}$ in the noiseless case. For evenly spaced data, the noise in the power spectrum is approximately $\overline{\delta m_{\mathrm{d}}} / K$, where $\overline{\delta m_{\mathrm{d}}}$ is the average photometric error and $K$ the number of points in the light curve. For a light curve with large occasional gaps, this result needs to be multiplied by a factor $1-\left(t_{\text {gaps }} / t_{\max }\right)$, where $t_{\max }$ is the total duration of the light curve and $t_{\text {gaps }}$ is the sum of the duration of the gaps. Peaks which are not more than several times this noise level should not be considered significant. Note that it is possible to detect a sinusoid of amplitude less than the photometric errors, because the noise is spread over many frequencies in the power spectrum.

We can reasonably search for sinusoidal periods up to the longest time span of the observations, $t_{\max }$, although if the coverage is very non-uniform then the sensitivity 
to the longer periods will be reduced. There is, in principle, information in the light curve on periods down to the smallest time separation between epochs. However, as the typical spacing between epochs is often more than this, the sensitivity at these very short periods is reduced. In Sect. 5 we search for periodicities between 0.4 hours and 125 hours (frequencies between $2.5 / \mathrm{hr}$ and $0.008 / \mathrm{hr}$ ). The uncertainty in a period is set by the finite resolution of the power spectrum. This is determined by the duration of the observations $\left(t_{\max }\right)$, which makes it impossible to distinguish between two closely separated frequencies, giving rise to an error in a period $\tau$ of $\tau^{2} /\left(2 t_{\max }\right)$ (Roberts et al. 1987). However, at very short periods, we place a lower limit on the temporal resolution due to the finite integration time.

It is useful to plot the light curve phased to any significant periods to ensure that similar variations are not seen in the reference stars. However, as will be seen in Sect. 6.2, the absence of sinusoidal variation in the target star does not mean that this is not a true periodicity. A more useful (but not foolproof) check of whether a periodicity is intrinsic to the target is to calculate the cleaned power spectrum of the reference stars. Strong peaks present in both this and the power spectrum of the target may not be intrinsic to the target. Note that this cannot be done reliably with the dirty power spectrum: we see from Eq. (7) that any "false" peaks in the dirty power spectrum, $D(\nu)$, are due to the convolution of the spectral window function, $W(\nu)$, with the true power spectrum, $G(\nu)$. While both target and reference stars have the same $W(\nu)$, they have different $G(\nu)$, so false peaks which appear in the dirty spectrum of the target will not necessarily be in the dirty spectrum of the reference stars. They should, however, both be absent in the cleaned spectra.

\subsection{Other methods}

We briefly investigated the phase dispersion minimization method of Cincotta et al. (1995) for detection of periodic variability. This method phases the light curve to a range of periods, and measures the appropriateness of the period using the Shannon information entropy in the amplitudephase space. Periodicities in the data give rise to minima of the information entropy. It was found that the most significant minima were due to the sampling, with dominant minima at 24 hours and rational multiples thereof. The numerous other minima were weak and obscured by noise. It seems that this method may not be suitable for time series with the relatively few number of points used here (Cincotta, private communication). This method has not, therefore, be pursued in any detail in this paper.

\section{Results}

\subsection{General results}

The results of the application of the $\chi^{2}$ test to the 21 targets are shown in Table 2 for the detections $(p<0.01)$ and Table 3 for the non-detections $(p>0.01)$ of variability. In these tables we use two measures of the variability amplitude. The first, $\sigma_{\mathrm{m}}$, is simply the rms (root-meansquare) value of $m_{\mathrm{d}}(k)$ for the whole light curve (all $k$ ). This measure disproportionately represents large values, so we also quote $\overline{\left|m_{\mathrm{d}}\right|}$, the mean of the absolute values of $m_{\mathrm{d}}(k)$. Assigning an amplitude in this low SNR regime is non-trivial. For example, using a slightly different aperture size can give a slightly different amplitude, because the noise changes. As the same aperture has been used for all objects (except SDSS 0539) these amplitudes are at least comparable. In general one needs to determine the amplitude by solving for a parametrized model, e.g. by marginalising over nuisance parameters in an appropriate Bayesian framework. We are not prepared to assign such a model at this time, so we simply report these measures.

For those objects in which we did not detect variability, we have set upper limits on the amplitude according to what we could have detected. This was done by creating a set of synthetic light curves by multiplying each $m_{\mathrm{d}}(k)$ by $1+a$, for increasing (small) values of $a$. The amplitude limits were obtained from that synthetic light curve which gave $p=0.01$ according to the $\chi^{2}$ test. (The $p$ value quoted in Table 3 is that from the actual data.)

The reliability of the $\chi^{2}$ test clearly depends on an accurate determination of the magnitude errors in the target. We have checked this by analysing the relative magnitude variations, $\overline{\left|m_{\mathrm{d}}\right|}$, in reference stars of similar brightness as the target (both before and after rejection of any variables). We found that these variations are similar to (and, in particular, no larger than) the mean error, $\overline{\delta m_{\mathrm{d}}}$, for the respective target, indicating that we are not underestimating the errors, and hence not overestimating $\chi^{2}$ or the significance of a detection.

We point out that the significance of a detection cannot be judged simply by looking at the ratio of $\sigma_{\mathrm{m}}$ to $\overline{\delta m_{\mathrm{d}}}$. This ratio is not the "sigma detection" level, because the light curve consists of many points: in the case of a very large number of epochs, statistically significant fluctuations could be recognised even if $\sigma_{\mathrm{m}}$ were hardly more than $\overline{\delta m_{\mathrm{d}}}$. The $\chi^{2}$ distribution takes this into account via the degrees of freedom.

Some of the detections/non-detections in the tables are close to the significance limit, for which a value of $p=0.01$ was chosen as being reasonably conservative. The choice is, however, somewhat arbitrary, and we could have chosen 0.05 or 0.001 , which would make some detections into nondetections, or vice versa. We mention this to emphasise that detections/non-detections close to the limit should be treated with due uncertainty.

Three of the detections in Table 2 (2M0913, 2M1146 and Calar 3) were non-detections in Paper I. These new detections have amplitudes below the limits placed on them in Paper I. Roque 11 and Teide 1 (non-detections in Paper I) remain non-detections, but now at lower amplitude limits. The increased sensitivity in the present paper come about for a number of reasons: improved flat fielding, including an illumination correction; better fringe 
Table 2. Variability detections. $t_{\max }$ is the maximum time span of observations: the minimum span was between 10 and 20 min. The amplitude of the observed variability is measured by the average (over all points in the light curve) of the absolute relative magnitudes, $\overline{\left|m_{\mathrm{d}}\right|}$, and the rms (root-mean-square) of the relative magnitudes, $\sigma_{\mathrm{m}} \cdot \overline{\delta m_{\mathrm{d}}}$ is the average photometric error in the light curve (also in magnitudes). $1-p$ is the probability that the variability is not compatible with the photometric errors. "Obs. run" refers to which of the three observing runs the data come from, in YYMM date format

\begin{tabular}{llrlrlrrrr}
\hline target & SpT & $\begin{array}{r}t_{\max } \\
\text { hours }\end{array}$ & $\overline{\left|m_{\mathrm{d}}\right|}$ & $\sigma_{\mathrm{m}}$ & $\overline{\delta m_{\mathrm{d}}}$ & $p$ & $\begin{array}{r}\text { No. } \\
\text { frames }\end{array}$ & $\begin{array}{r}\text { No. } \\
\text { refs }\end{array}$ & $\begin{array}{r}\text { Obs. } \\
\text { run }\end{array}$ \\
\hline 2M0345 & L0 & 53 & 0.012 & 0.017 & 0.011 & $4 \mathrm{e}-4$ & 27 & 23 & $99-09$ \\
2M0913 & L3 & 125 & 0.042 & 0.055 & 0.039 & $7 \mathrm{e}-4$ & 36 & 14 & $99-01$ \\
2M1145 & L1.5 & 124 & 0.026 & 0.031 & 0.022 & $1 \mathrm{e}-3$ & 31 & 12 & $99-01$ \\
\multicolumn{1}{l}{} & " & 76 & 0.015 & 0.020 & 0.012 & $<1 \mathrm{e}-9$ & 70 & 11 & $00-02$ \\
2M1146 & L3 & 124 & 0.012 & 0.015 & 0.011 & $3 \mathrm{e}-3$ & 29 & 7 & $99-01$ \\
2M1334 & L1.5 & 126 & 0.017 & 0.020 & 0.011 & $<1 \mathrm{e}-9$ & 51 & 12 & $00-02$ \\
SDSS 0539 & L5 & 76 & 0.009 & 0.011 & 0.007 & $3 \mathrm{e}-5$ & 31 & 24 & $00-02$ \\
SDSS 1203 & L3 & 52 & 0.007 & 0.009 & 0.007 & $2 \mathrm{e}-3$ & 51 & 13 & $00-02$ \\
Calar 3 & M9 & 29 & 0.026 & 0.035 & 0.027 & $6 \mathrm{e}-4$ & 42 & 21 & $99-01$ \\
S Ori 31 & (M6.5) & 50 & 0.010 & 0.012 & 0.007 & $4 \mathrm{e}-5$ & 21 & 30 & $00-02$ \\
S Ori 33 & (M6.5) & 51 & 0.008 & 0.010 & 0.007 & $2 \mathrm{e}-3$ & 21 & 43 & $00-02$ \\
S Ori 45 & M8.5 & 50 & 0.051 & 0.072 & 0.032 & $5 \mathrm{e}-9$ & 21 & 30 & $00-02$ \\
\hline
\end{tabular}

Table 3. Variability non-detections. The columns are the same as in Table 2 except that here $\overline{\left|m_{\mathrm{d}}\right|}$ and $\sigma_{\mathrm{m}}$ are the upper detection limits on the variability amplitudes. The minimum time between observations of a given target was between 3 min (for 2M1439) and 35 min (for Roque 12)

\begin{tabular}{llrlcccrrr}
\hline target & SpT & $\begin{array}{r}t_{\max } \\
\text { hours }\end{array}$ & $\overline{\left|m_{\mathrm{d}}\right|}$ & $\sigma_{\mathrm{m}}$ & $\overline{\delta m_{\mathrm{d}}}$ & $p$ & $\begin{array}{r}\text { No. } \\
\text { frames }\end{array}$ & $\begin{array}{r}\text { No. } \\
\text { refs }\end{array}$ & $\begin{array}{r}\text { Obs. } \\
\text { run }\end{array}$ \\
\hline 2M0030 & L2 & 51 & 0.018 & 0.025 & 0.020 & 0.21 & 37 & 27 & $99-09$ \\
2M0326 & L3.5 & 49 & 0.021 & 0.029 & 0.017 & 0.56 & 19 & 36 & $99-09$ \\
2M1439 & L1 & 97 & 0.007 & 0.009 & 0.007 & 0.10 & 48 & 13 & $00-02$ \\
Roque 11 & M8 & 100 & 0.028 & 0.043 & 0.027 & 0.46 & 47 & 23 & $99-01$ \\
Roque 12 & M7.5 & 50 & 0.016 & 0.022 & 0.015 & 0.02 & 17 & 43 & $99-09$ \\
Roque 16 & M6 & 29 & 0.010 & 0.014 & 0.010 & 0.35 & 16 & 34 & $99-09$ \\
Teide 1 & M8 & 100 & 0.029 & 0.041 & 0.030 & 0.10 & 47 & 23 & $99-01$ \\
S Ori 34 & (M6) & 51 & 0.008 & 0.010 & 0.007 & 0.28 & 21 & 43 & $00-02$ \\
S Ori 44 & M6.5 & 51 & 0.030 & 0.035 & 0.026 & 0.06 & 21 & 30 & $00-02$ \\
S Ori 46 & (M8.5 & 51 & 0.032 & 0.041 & 0.030 & 0.03 & 21 & 43 & $00-02$ \\
\hline
\end{tabular}

removal, including a lower noise fringe correction image; use of more reference stars; a slightly smaller photometry aperture to improve the SNR.

In analysing the light curves, it came to our attention that four of the five targets from 99-09 seem to have a lower average flux on the last (fourth) night than the average of the preceding three nights (by 0.01 to 0.03 magnitudes; the fifth target, Roque 12 , was not observed on this night). This effect is not seen, however, in any of the reference stars, not even ones of similar brightness to the targets. After eliminating other potential problems with the observing and reduction, one possible cause is that the effective bandpass was different on this night. As the reference stars are presumably much bluer on average than the targets, this could change the magnitude of the targets relative to the reference stars without changing the magnitudes of the reference stars relative to one another. The beginning of the fourth night was lost due to clouds and humidity, and residual cloud cover could have remained for the rest of the night. However, it appears that a thin cloud layer does not significantly alter the wavelength dependence of the atmospheric extinction coefficient over the $I$ band (Driscoll 1978), so we cannot provide a satisfactory explanation of this observation at this time. Although it is possible that the effect is intrinsic to all four objects, it is rather suspicious, so we exclude this night from our analysis and the results presented in the tables. If this night were included, 2M0030 and Roque 16 would become detections. No such correlated behaviour is seen in the targets from the other runs. Broad band differential photometry can be affected by second-order colour dependent extinction, even in clear conditions, but this 


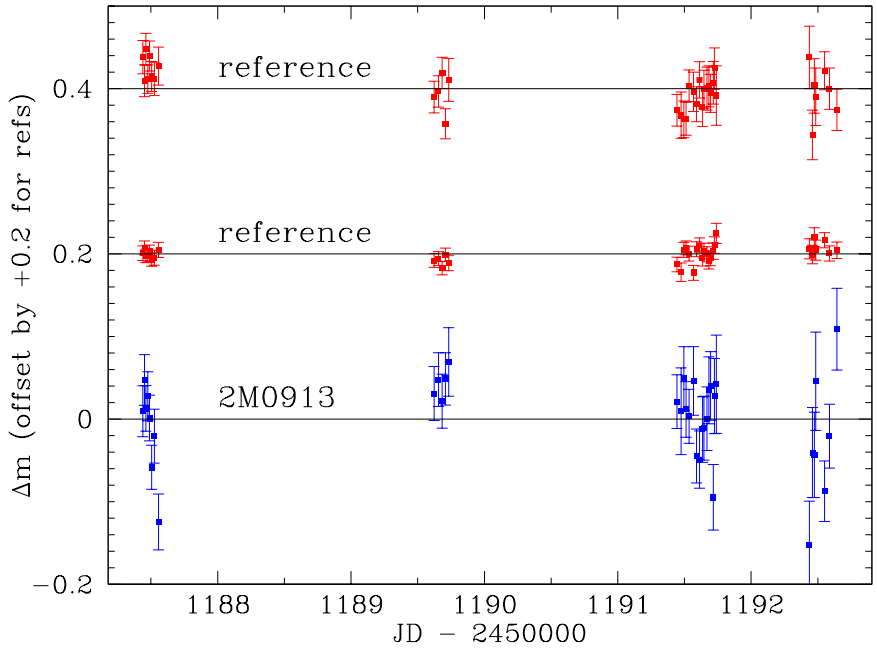

Fig. 1. Light curve for $2 \mathrm{M} 0913$ (bottom). Plotted above this for comparison are a reference star of similar magnitude (top) and a bright reference star (middle). The mean of each light curve is shown with a solid line. The light curves for the two reference objects are offset from that for the target star by the amount shown on the vertical axis

contribution is estimated to be well below the $0.5 \%$ error (see Young 1991 for a discussion).

\subsection{Comments on individual objects}

Notes are now given on all the objects with statistically significant $\chi^{2}$ detections, along with brief comments at the end of the section on the non-detections. The implications of these results will be discussed in Sect. 6 .

2M0345. The light curve shows no interesting features and there are no peaks in the cleaned power spectrum above four times the noise. If the dubious fourth night is included this becomes a very significant detection $(p<1 \mathrm{e}-9)$.

2M0913. This detection is due primarily to a significant drop in the flux around AJD 1187.5 (Fig. 1), going down to 0.13 magnitudes below the median for that night, and can be seen when a range of aperture sizes are used for the photometry. Although there was some cloud and Moon around this time, no similar drop is seen in the reference stars, including two of similar brightness to 2M0913. Furthermore, two other targets taken at this time (2M1145 and 2M1146) do not show this behaviour. There is no evidence for variability within the other three nights. There are no strong periodicities in the cleaned power spectrum, the strongest three being at 3.36, 0.76 and $0.64( \pm 0.08)$ hours, each at around only five times the noise level.

2M1145. Evidence for variability in this L dwarf was presented in Paper I, and it was tentatively claimed to be periodic with a period of 7.1 hours (using the Lomb-Scargle periodogram), pending confirmation. The cleaned power

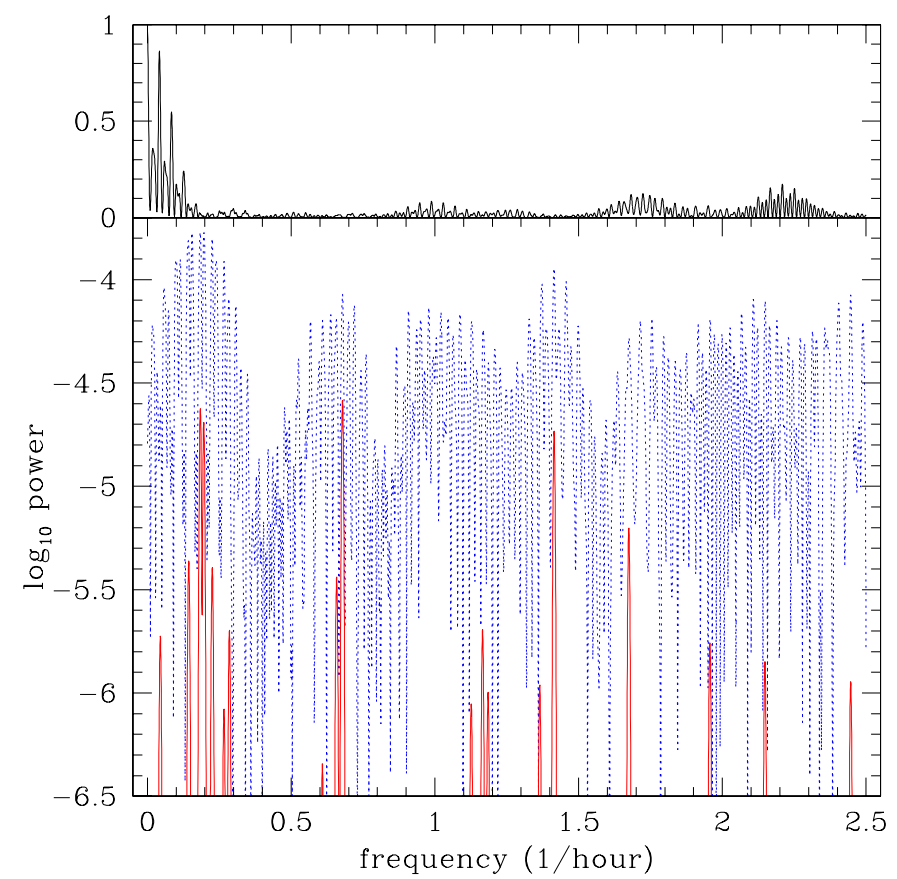

Fig. 2. Power spectrum for $2 \mathrm{M} 1145$ light curve from the 9901 run. The bottom panel shows the dirty spectrum (dotted line) and the cleaned spectrum (solid line) in units of $\log _{10}(P)$. The noise level is at about $\log _{10}(P)=-5.6$. The top panel shows the spectral window function on a linear vertical scale, normalised to a peak value of 1.0

spectrum of these same data (old reduction) gives peaks at $7.1 \pm 0.3$ and $0.71 \pm 0.08$ hours. The new reduction of these data still gives evidence for variability, but the cleaned power spectrum shows peaks (all at about eight times the noise) at $5.4 \pm 0.1,5.1 \pm 0.1,1.47 \pm 0.08$ and $0.71 \pm 0.08$ hours (Fig. 2).

The improved reduction in the present paper has reduced the average photometric error from 0.027 to 0.022 magnitudes. (Three additional frames in the new reduction two nights earlier are also used, which improves the resolution of the power spectrum.) The light curves from the two reductions are consistent within their combined errors. A small peak is still seen around 7.1 hours in the new reduction, but it has far less power. In the dirty spectrum, this peak is one of the strongest, indicating that it has probably been artificially enhanced by the window function: this demonstrates the necessity of cleaning the power spectrum. We see in Fig. 2 how difficult it would be to confidently locate the dominant peaks in the dirty spectrum. We are confident of the superiority of the new reduction, so while the variability detection in $2 \mathrm{M} 1145$ in Paper I still holds, the tentatively assigned period of 7.1 hours does not.

2M1145 was re-observed at higher SNR and with more epochs across four nights in the 00-02 run. These data (Fig. 3) also show very strong evidence for variability, and the power spectrum shows four significant peaks at the following periods (with power in units of the noise in parentheses): $11.2 \pm 0.8$ (31), $6.4 \pm 0.3$ (14), 


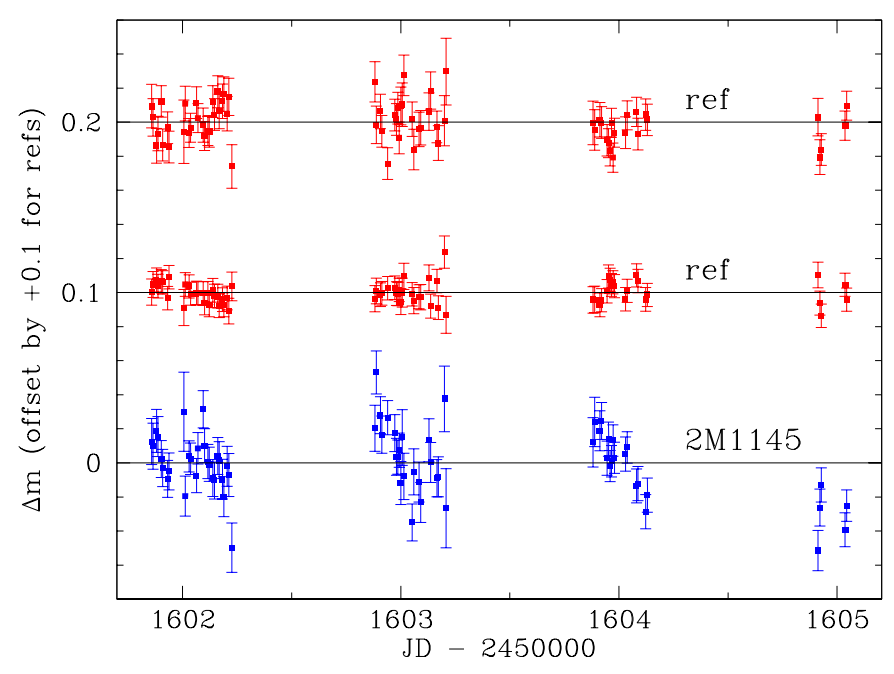

Fig. 3. Light curve for $2 \mathrm{M} 1145$ from the 00-02 run (bottom) plus a bright reference object (middle) and one of similar brightness to the target (top). See caption to Fig. 1

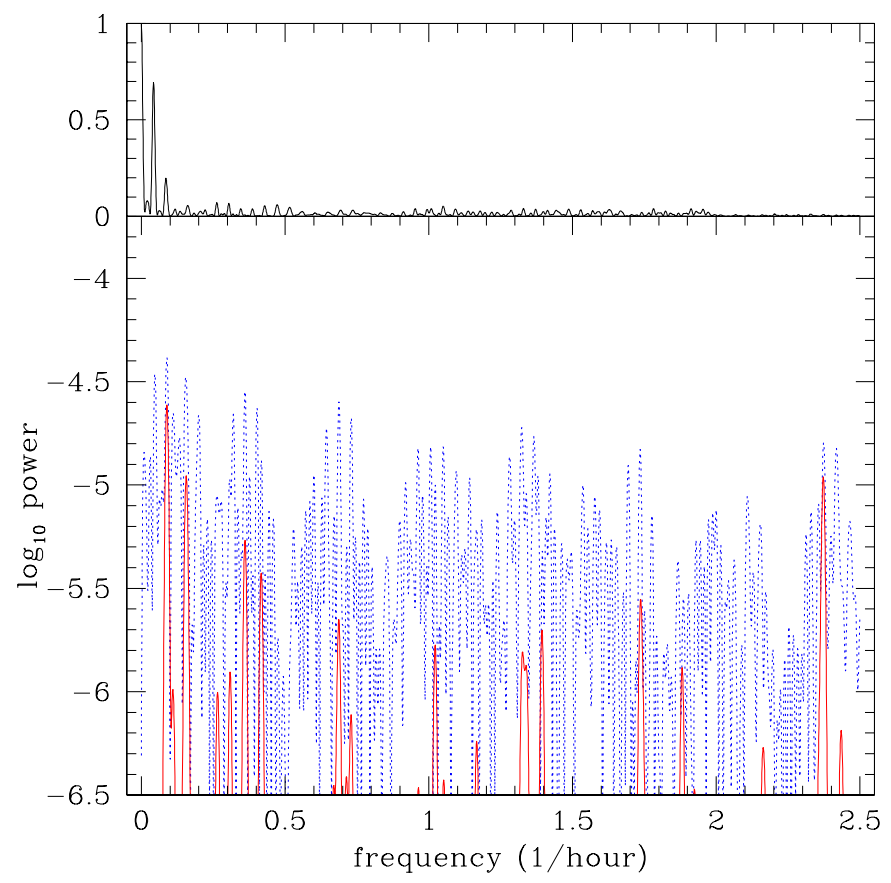

Fig. 4. Power spectrum for $2 \mathrm{M} 1145$ (from 00-02). The noise level is $\log _{10}(P)=-6.1$. See caption to Fig. 2

$2.78 \pm 0.13$ (7), $0.42 \pm 0.13$ (14) hours (Fig. 4). Note that the first period is four times the third, so these may not be independent. There are essentially no common peaks in this power spectrum and the one from 99-01. As mentioned earlier, most epochs in the 00-02 run were taken in pairs with no time gap between them. This enables us to produce a binned light curve consisting of 33 points (four single points removed). The cleaned power spectrum of this only has a significant periodicity at $11.3 \pm 0.8$ hours ( 8 times the noise). There is still a periodicity at $2.77 \pm 0.30$ hours, but now at only five times the noise level. It is unlikely that either of these is the rotation period because neither was detected in the

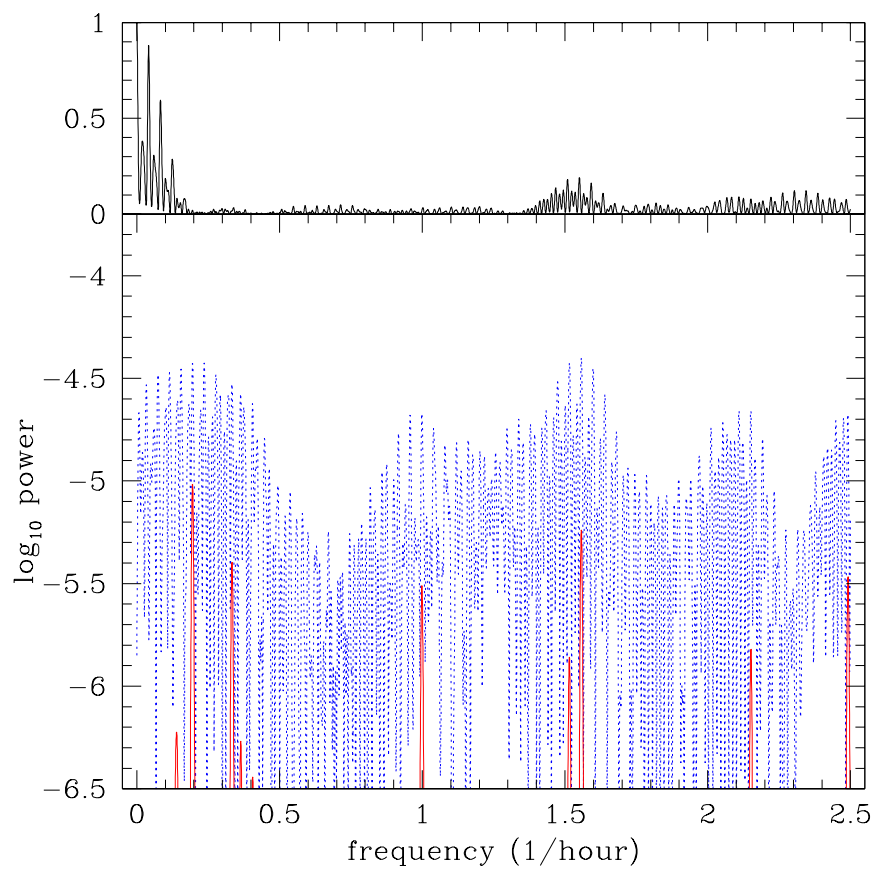

Fig. 5. Power spectrum for $2 \mathrm{M} 1146$. The noise level is $\log _{10}(P)=-6.1$. See caption to Fig. 2

99-01 data (Fig. 2). We can be confident that 2M1145 does not have both stable (over a one year timescale) surface features and a rotation period of between 1 and 70 hours. If it did, we would have detected such a rotation period in both runs (see Sect. 6.2).

2M1146. This is a marginal detection which was a marginal non-detection in the original reduction. The power spectrum shows peaks at the following periods (with power in units of noise): $5.1 \pm 0.1$ (15), $3.00 \pm 0.08$ (6), $1.00 \pm 0.08$ (5), and $0.64 \pm 0.08$ (9) hours (Fig. 5). The second and third are in the ratio $3: 1$, so are probably not independent. The one at three hours is more convincing based on the phase coverage in the phased light curve. This is one of only two $\mathrm{L}$ dwarfs in our sample which already has a measured $v \sin i$ of $32.5 \pm 2.5 \mathrm{~km} \mathrm{~s}^{-1}$ (Basri et al. 2000). For an object of radius $0.1 R_{\odot}$ (expected for these objects, see Chabrier \& Baraffe 2000), this implies a rotation period of $3.7 \pm 0.3$ hours, or less, due to the unknown inclination, $i$, of the rotation axis to the line of sight. In the case of 2M1146, however, there is another complicating factor, namely that Koerner et al. (1999) have observed it to be a brightness ratio one binary, with separation $0.3^{\prime \prime}$ $(7.6 \mathrm{AU})^{7}$. This was not resolved by our observations, so our light curve (and power spectrum) is a composite of the two objects. It is possible, therefore, that two of the three peaks in the power spectrum are rotation periods for the objects. Kirkpatrick et al. (1999) also

\footnotetext{
7 The circular orbital speed about their centre-of-mass is less than $2.5 \mathrm{~km} \mathrm{~s}^{-1}$ (assuming masses of $<0.1 M_{\odot}$ ), so does not complicate the $v \sin i$ determination of Basri et al.
} 


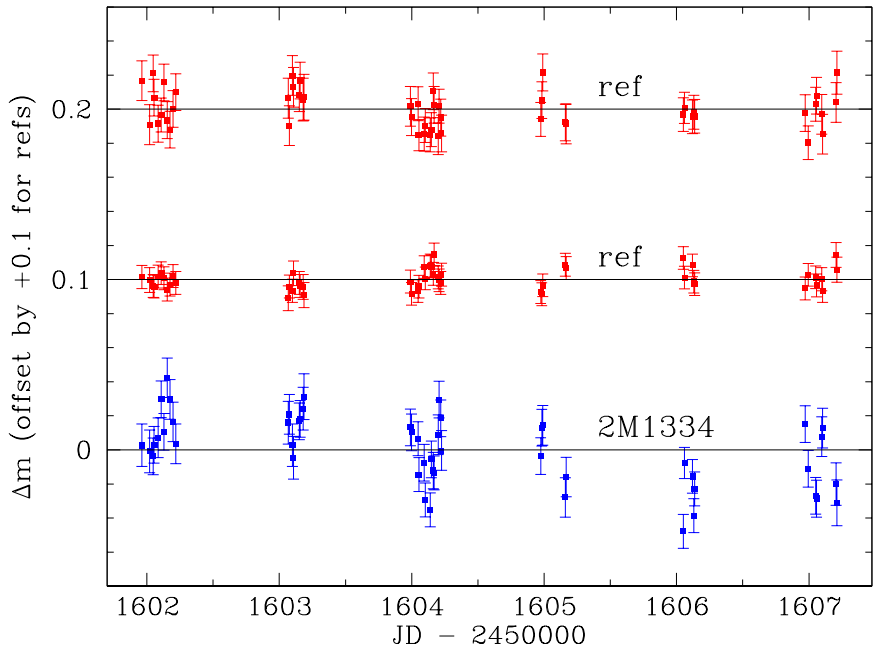

Fig. 6. Light curve for 2M1334 (bottom) plus a bright reference object (middle) and one of similar brightness to the target (top). See caption to Fig. 1

found an earlier type star $1^{\prime \prime}$ away, which is presumably a background star, and this too could affect our light curves.

2M1334. This is significantly variable, and the light curve shows clear fluctuations within a number of nights (Fig. 6). The largest peak in the power spectrum (Fig. 7) is at $2.68 \pm 0.13$ hours at 12 times the noise. If we look more closely at the raw light curve, the first three nights would appear to show a periodicity on the scale of a few hours (the $\chi^{2}$ value for just these three nights is $p=2 \mathrm{e}-6)$. The power spectrum of just these three nights shows peaks at $6.3 \pm 0.4$ and $1.01 \pm 0.08$ hours at six and seven times the noise respectively.

Calar 3. The light curve (Fig. 8) does not look qualitatively different from that of three reference stars of similar brightness, apart from some "activity" around AJD $=1191.5$. The two most significant peaks in the power spectrum (at 14.0 and 8.5 hours) are less than five times the noise level, so are barely significant.

SDSS 0539. The seeing was worse than average for many of the frames in this field, so a larger photometry aperture of radius 5.0 pixels was used. (Use of a bigger aperture generally decreases the significance of a detection as it increases the noise, so using a larger aperture in this case is more conservative.) The significant $\chi^{2}$ is partly due to the brighter points around AJD 1604. Otherwise the light curve shows no obvious patterns (see Fig. 9). The power spectrum shows a significant (20 times noise) peak at $13.3 \pm 1.2$ hours (Fig. 10). The light curve phased to this period is shown in Fig. 11.

SDSS 1203. This variability is primarily due to a drop in brightness of about 0.02 magnitudes in four consecutive measurements around AJD = 1606.1 (Fig. 12). The drop lasts between one and two hours. Particularly interesting

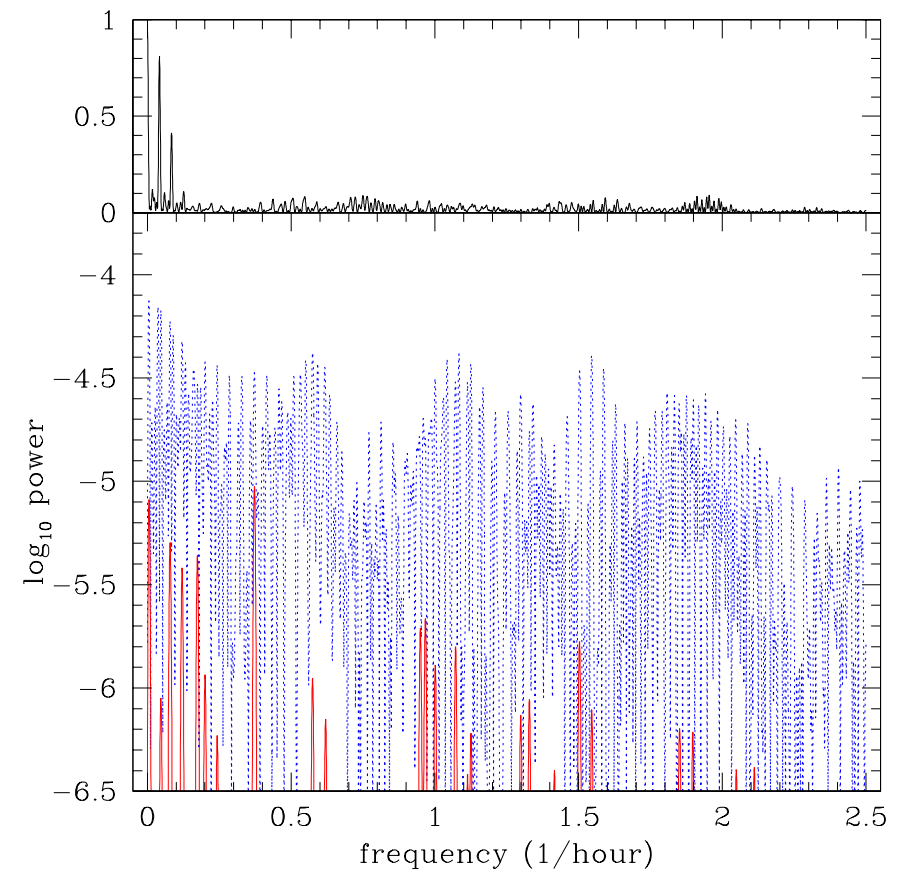

Fig. 7. Power spectrum for 2M1334 (all nights). The noise level is $\log _{10}(P)=-6.2$. See caption to Fig. 2

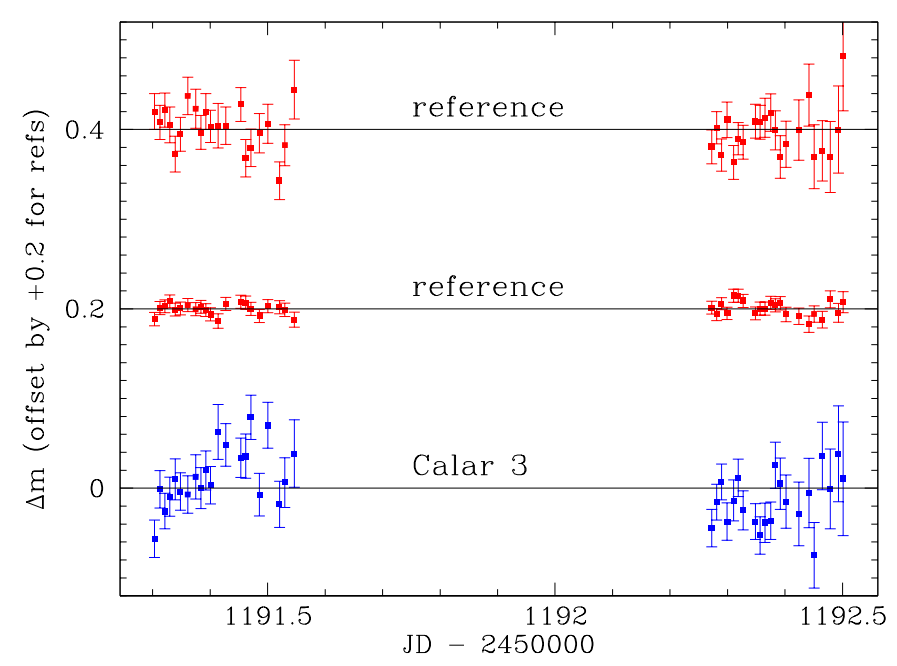

Fig. 8. Light curve for Calar 3 (bottom) plus a bright reference object (middle) and one of similar brightness to the target (top). See caption to Fig. 1

here (as drops in a few consecutive points are often seen) is that the light curve never drops this low at any other time. It could be attributed to an eclipse by a physically associated companion. This would either have to be very close or of much lower luminosity and hence mass, possibly a planetary companion. There are of course other explanations, such as a short-lived surface feature.

S Ori 31. The light curve and power spectrum are shown in Figs. 13 and 14. The latter shows two significant peaks at $7.5 \pm 0.6$ and $1.75 \pm 0.13$ hours at 18 and 9 times the noise level respectively. The former period dominates and shows reasonable evidence for sinusoidal variation 


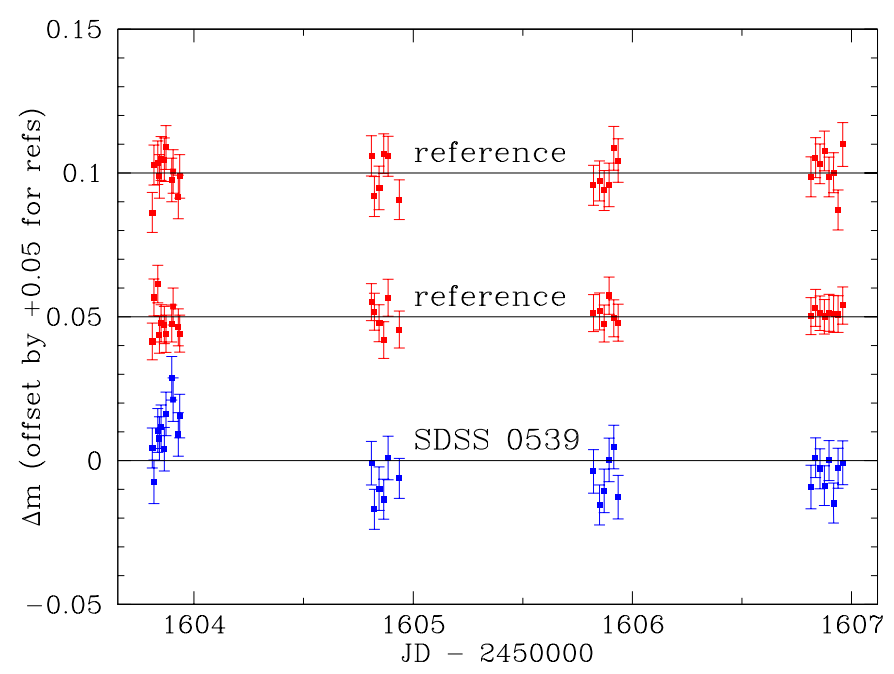

Fig. 9. Light curve for SDSS 0539 (bottom) plus a bright reference object (middle) and one of similar brightness to the target (top). See caption to Fig. 1

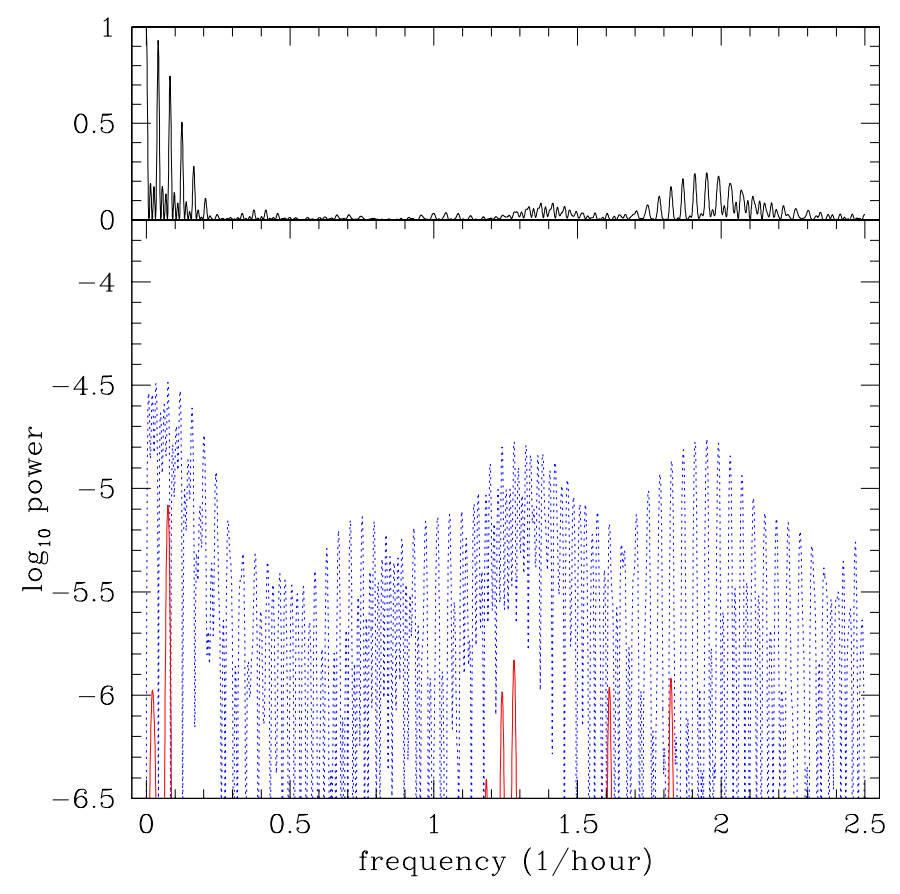

Fig. 10. Power spectrum for SDSS 0539. The noise level is $\log _{10}(P)=-6.4$. See caption to Fig. 2

(Fig. 15), with an amplitude of about 0.01 magnitudes, and may be the rotation period for this object.

S Ori 33. The light curve (Fig. 16) shows a rise just before AJD 1606, and the power spectrum (Fig. 17) has peaks of 6 to 7 times the noise at $8.6 \pm 0.7$ and $6.5 \pm 0.4$ hours. Although neither is very significant, the phased light curve at 8.6 hours shows reasonable sinusoidal variation (Fig. 18) with an amplitude of around 0.015 magnitudes. This could be the rotation period. The

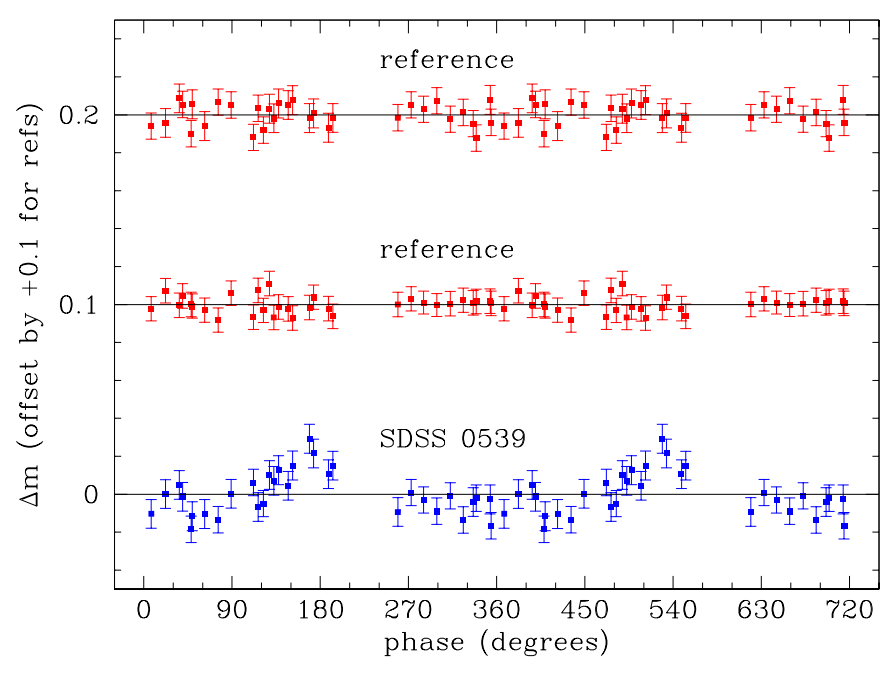

Fig. 11. Light curve (bottom) for SDSS 0539 phased to a period of 13.3 hours. The cycle is shown twice (labelled $0^{\circ}-360^{\circ}$ and $360^{\circ}-720^{\circ}$ ). This phased light curve (plus all others in this paper) has been plotted to have the same phase as a sine wave. Also shown are two reference stars phased in the same way

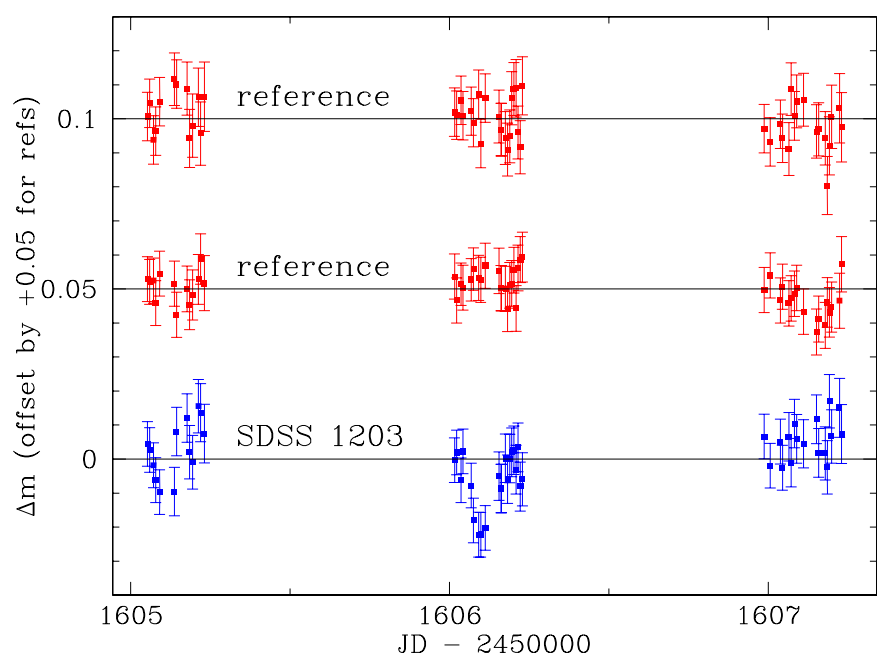

Fig. 12. Light curve for SDSS 1203 (bottom) plus a bright reference object (middle) and one of similar brightness to the target (top). See caption to Fig. 1

light curve phased to 6.5 hours, on the other hand, gives a much poorer fit to a sine wave.

S Ori 45. The light curve shows three points much lower than the average around AJD 1604.9. Indeed, the five points on this first night of observations span a range of almost 0.25 magnitudes. If these points are excluded there is no evidence for variability $(p=0.18)$. There is a bright $(\Delta m=1.7)$ star nearby $\left(5^{\prime \prime}\right)$ which may well interfere with this variability determination. For example, small changes in this star's brightness could result in large changes in the apparent brightness of S Ori 45 due to the flux gradient across the sky and photometry apertures of S Ori 45 . The most significant 


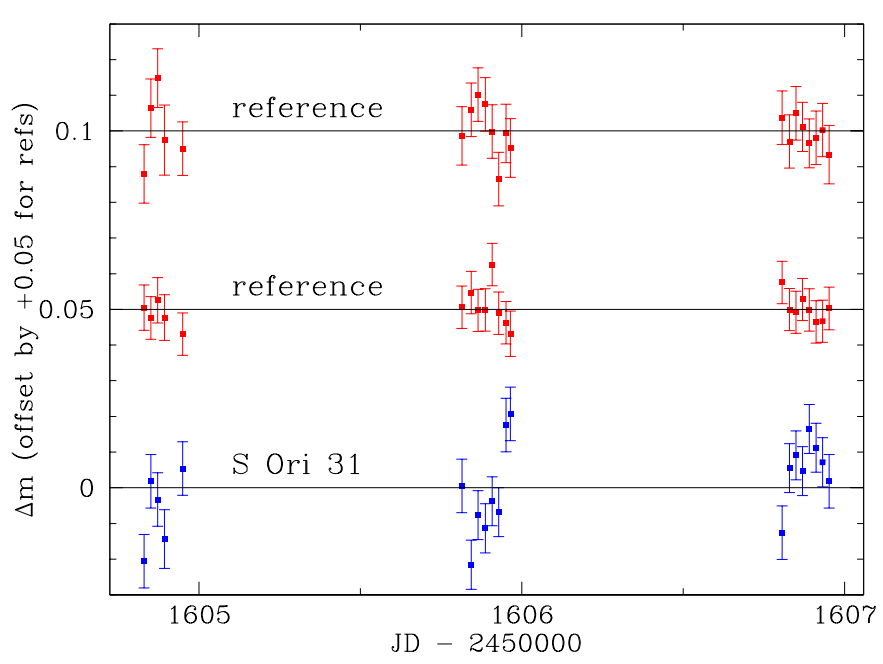

Fig. 13. Light curve for S Ori 31 (bottom) plus a bright reference object (middle) and one of similar brightness to the target (top). See caption to Fig. 1

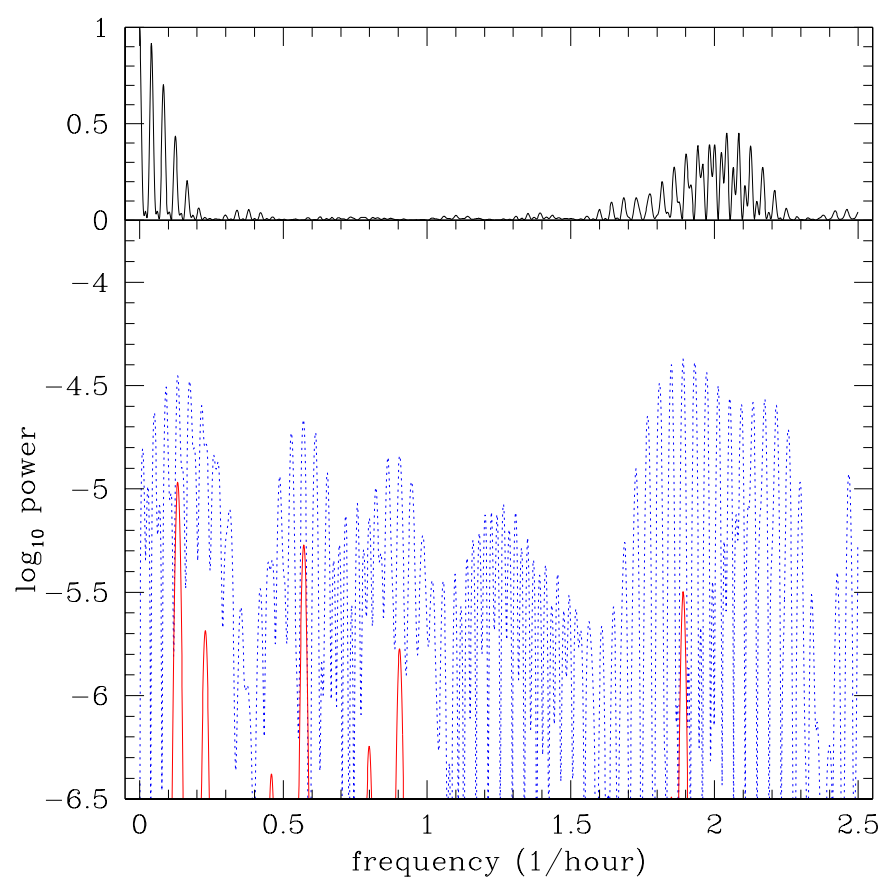

Fig. 14. Power spectrum for $\mathrm{S}$ Ori 31. The noise level is $\log _{10}(P)=-6.2$. See caption to Fig. 2

peak in the power spectrum is at $0.50 \pm 0.13$ hours (at 20 times the noise), which would be extremely fast if it is the rotation period. Clearly, much more rapid monitoring is required to determine this. There is a dip in three points around AJD 1606.9, similar to that seen in SDSS 1203, but we are hesitant to draw conclusions given the proximity of the bright star. S Ori 44 was observed in the same frame as S Ori 45 , and if we plot the light curve of one relative to the other, we see that $m_{\text {sori44 }}-m_{\text {sori45 }}$ varies between +0.15 and -0.18 mag with a mean of -0.05 and a standard error in this mean of 0.01 mag. This is interesting, as Béjar et al. (1999)

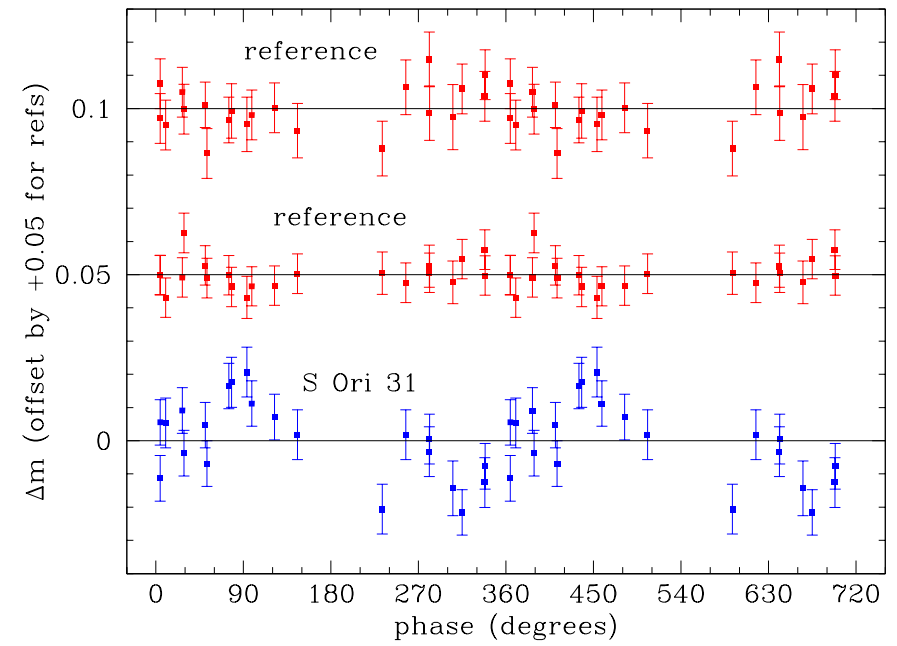

Fig. 15. Light curve (bottom) for S Ori 31 phased to a period of 7.5 hours. Also shown are the two reference stars from Fig. 13

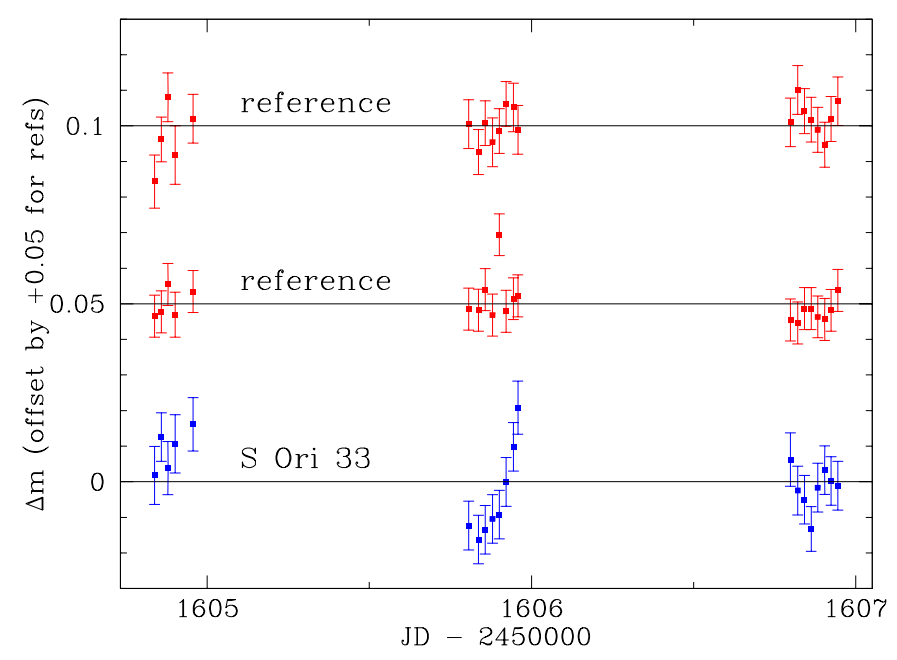

Fig. 16. Light curve for S Ori 33 (bottom) plus a bright reference object (middle) and one of similar brightness to the target (top). See caption to Fig. 1

give $m_{\text {sori44 }}-m_{\text {sori45 }}=-0.20 \pm 0.08$ mag. While these values are not inconsistent, the discrepancy could support evidence for variability in at least one of the objects.

Non-detections. $2 \mathrm{M} 1439$ has been measured by Basri et al. (2000) to have a $v \sin i$ of $10 \pm 2.5 \mathrm{~km} \mathrm{~s}^{-1}$, implying a period of less than 12.1 hours for a $0.1 R_{\odot}$ radius. S Ori 44 shows three consecutive points around AJD 1605.9 lower than the other five points on that night by about 0.09 magnitudes, possibly indicative of an eclipse, but unlike SDSS 1203 the $\chi^{2}$ is not significant (the errors are much larger for S Ori 44), and on the following night there are several points at this level. S Ori 46 has a bright nearby star, which may affect our attempt to determine variability. Roque 11 and Teide 1 have also been observed for variability in the $I$ band by Terndrup et al. (1999). They also did not find evidence for variability, with measured values of $\sigma_{\mathrm{m}}$ (rather than detection limits) of 0.041 and 0.045 magnitudes respectively. 


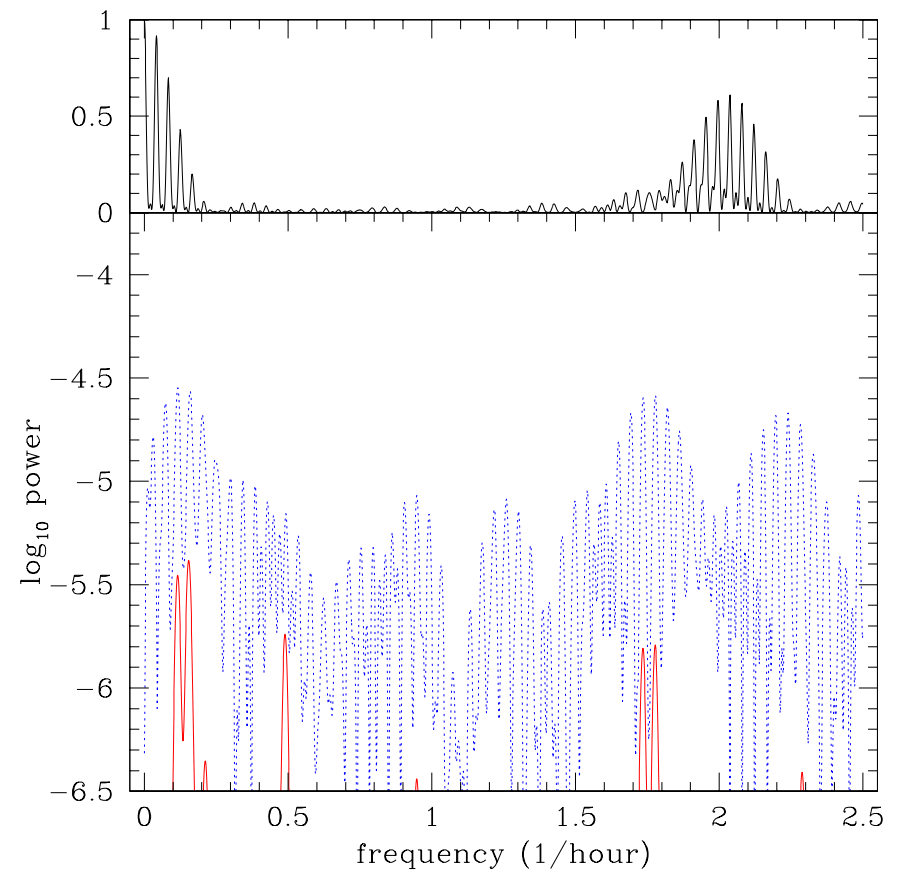

Fig. 17. Power spectrum for S Ori 33. The noise level is $\log _{10}(P)=-6.2$. See caption to Fig. 2

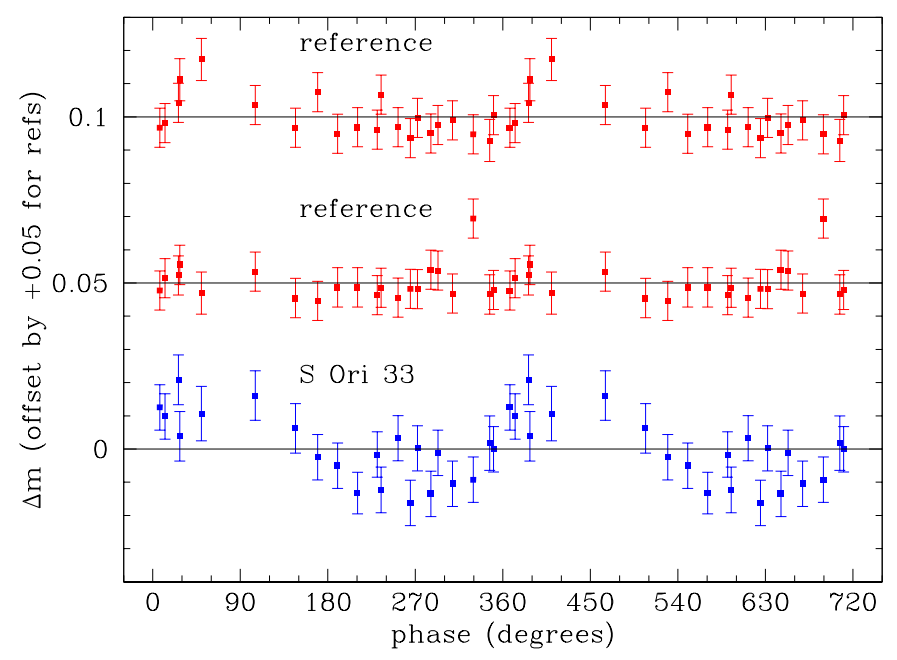

Fig. 18. Light curve (bottom) for S Ori 33 phased to a period of 8.6 hours. Also shown are the two reference stars from Fig. 16

\section{Discussion}

\subsection{General comments}

Of the 21 targets observed, 11 show evidence for variability at the $99 \%$ confidence level $(p=0.01)$. Of these, four (2M1145, 2M1334, SDSS 0539, S Ori 31) show strong evidence for variability $(p<1 \mathrm{e}-4)$. S Ori 45 is formally a fifth object with strong evidence for variability, but the presence of a bright close star makes us hesitant to draw this conclusion. In four cases (2M1146, 2M1334, SDSS 0539, S Ori 31) we have detected dominant significant periods in the range 3-13 hours, which may be rotation periods in all but the first case. S Ori 45 also has a dominant peak, but at 0.5 hours this would be very rapid if it is a rotation. The remaining objects do not show dominant periods, although the two earliest-type variables (S Ori 31 and S Ori 33) show near-sinusoidal light curves at detected periods. The light curve of one object, SDSS 1203, is essentially featureless except for a dip which may be due to an eclipse by a companion, although there is no direct evidence for this.

All of the objects which show variability have rms amplitudes $\left(\sigma_{\mathrm{m}}\right.$ in Table 2$)$ between 0.01 and 0.055 magnitudes (ignoring $\mathrm{S}$ Ori 45 ). The lower limit is set by the sensitivity of the observations, but no such upper limit is set. Thus one conclusion from this work is that these objects generally only have small amplitude variations, most in the range 0.01 to 0.03 magnitudes, on timescales of typically a few to a few tens of hours. The large fraction of non-detections (50\%), with upper limits on their rms amplitudes as low as 0.01 magnitudes, indicates that at least some ultra cool dwarfs have variability amplitudes less than 0.01 magnitudes.

These detections/non-detections are claimed on the basis of a $\chi^{2}$ test of the light curves. This requires a careful estimation of the photometric errors for the target objects: we confirmed that these were not underestimated via a comparison with the variability level of stars in the field of similar brightness. Additionally, the use of many reference stars (from which variables were first eliminated), plus the conservative assignment of a flat-fielding and fringe-removal error, gives us good confidence that we have not overestimated the significance of detections. We highlight that the $99 \%$ confidence level for the detection of variability is a somewhat arbitrary one: the division between Tables 2 and 3 represents a confidence level and not a definitive statement of what is and what is not variable at a certain amplitude.

\subsection{Rotation and surface features: Simulations}

The power spectrum is a representation of the light curve in the frequency domain (Eq. 6): $P(\nu)$ is the contribution of a sinusoid at frequency $\nu$ to the variance in the light curve $g(t)$. The goal of this analysis is to see whether the light curve can be more simply explained in this domain. However, the presence of a significant peak in the power spectrum does not mean that this is a long-term periodicity. After all, any light curve, including a random one, can be described in terms of its power spectrum, so the features in the light curve must appear somewhere in the power spectrum. The question is whether this description tells us anything useful about the source. If we detect just one or two dominant peaks then it may well be appropriate to describe the light curve as periodic at the detected period(s). If, on the other hand, we detect a large number of peaks, then, given that we have a finite number of data points, these peaks are less likely to correspond to true long-term periodicities.

The ideal case of a pure sinusoidal light curve is only produced by a rotating star if one hemisphere is uniformly 

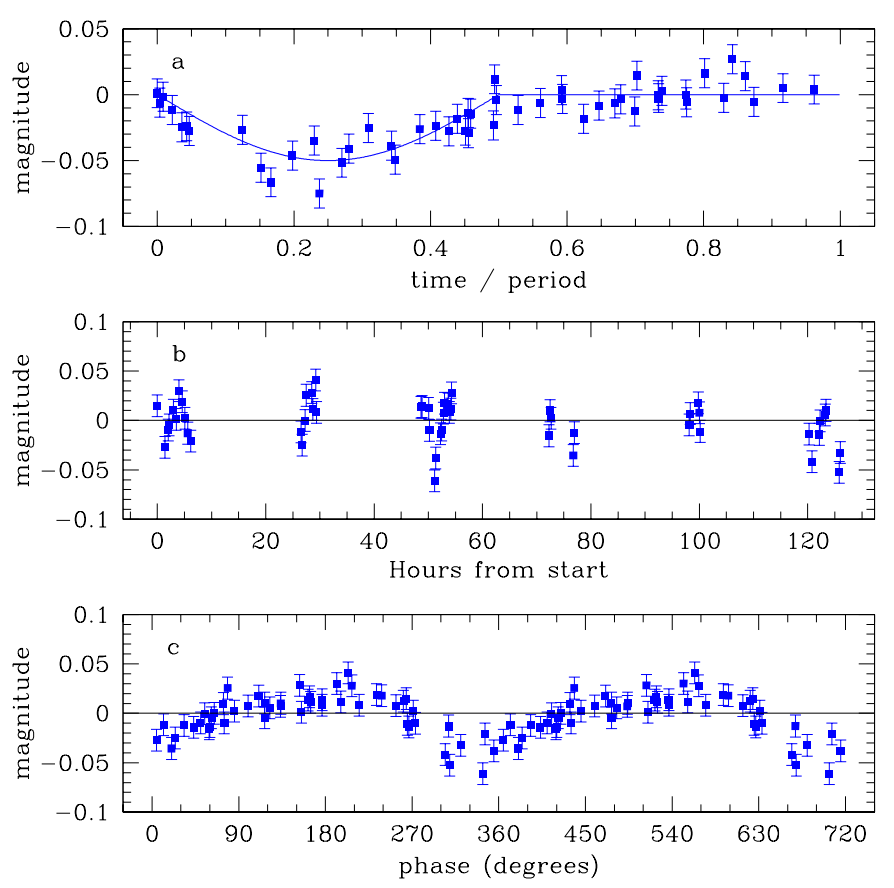

Fig. 19. Simulation of the light curve of a spotted rotating star. a) The solid line shows the true (noiseless) light curve of rotating star viewed equatorially with a single dark spot which causes a dimming of a maximum of 0.05 magnitudes. If the rotation period is five hours and the star is observed in the same way as $2 \mathrm{M} 1334$, i.e. with the same time sampling and Gaussian noise with standard deviation of 0.011 magnitudes, we obtain the light curve in $\mathbf{b}$ ), which, when wrapped to the rotation period gives the points plotted in a). This is significantly variable according to the $\chi^{2}$ test $(p<1 \mathrm{e}-9)$. A CLEAN power spectral analysis of this light curve (Fig. 20) reveals a period of $5.01 \pm 0.10$ hours: the light curve phased to this detected period and phase is shown in c) (cycle shown twice)

darker than the other and the star is observed along its equatorial plane. A star with a single small surface feature ("spot") would show a sinusoidal pattern (due to a cosine projection effect) only when the spot is on the observable hemisphere; for up to half of the rotation (depending on the inclination of the rotation axis) the light curve would be constant. A star with two spots would show a yet more complex light curve, as two, one or no spots are observable at any one time. While these light curves will be periodic, they will not be sinusoidal, as additional sine waves are required to reconstruct the exact shape of the light curve. Hence the power spectrum of the light curve of a rotating star will typically consist of several peaks, any number of which may be significant. Of course, certain spot patterns may give rise to near-sinusoidal variations, but not necessarily so. For example, several of the light curves of Herbst et al. (2001) are periodic but not due to a single sinusoidal component.

We have simulated the appearance of the light curves in a few such situations. Figure 19 shows the light curve due to a single small dark spot on a star which causes a maximum 0.05 magnitude decrease in brightness. If we rotate this star with a period of five hours and observe

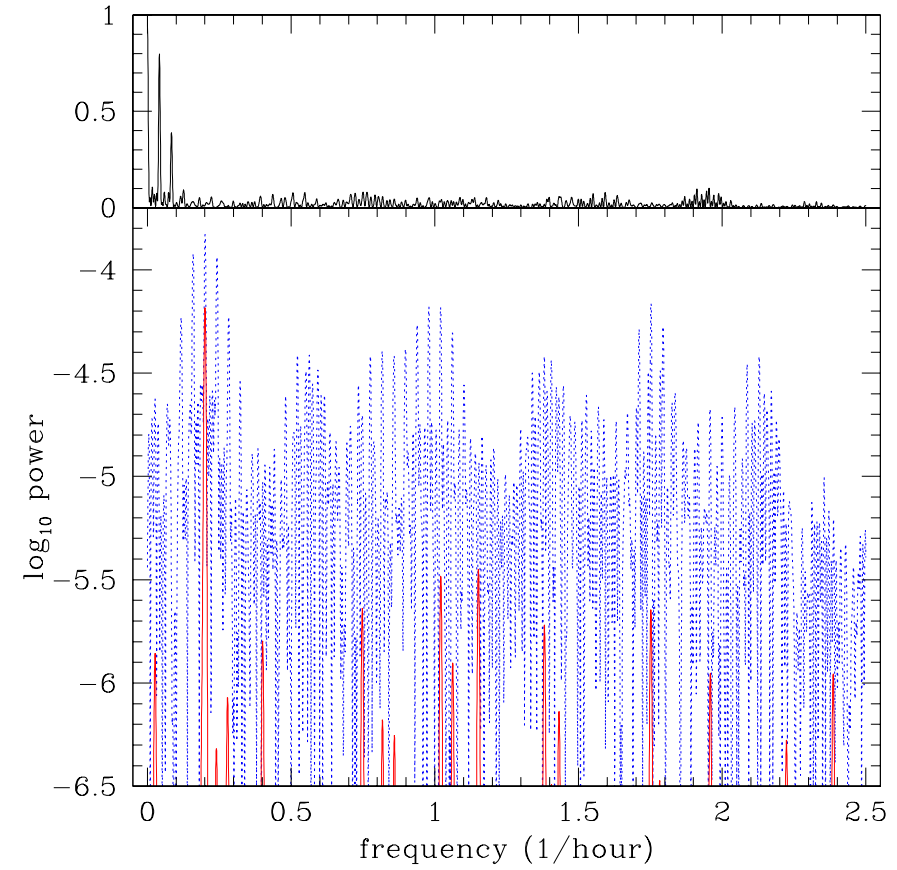

Fig. 20. Power spectrum for the simulated light curve shown in Fig. 19b. The noise level is $\log _{10}(P)=-6.2$. The same CLEAN parameters were used here as for the real data of Sect. 5. See caption to Fig. 2

it with the same noise level and time sampling as one of our target objects (2M1334) we obtain the power spectrum and phased light curve in Figs. 20 and 19c respectively. We see that the power spectrum picks out the rotation period despite the noise and despite the fact that the light curve is not sinusoidal. Furthermore, the phased light curve certainly does not resemble a sine wave, yet this is the rotation period. Another example is shown in Fig. 21 where we now have five small dark spots with random longitudes (i.e. phases) causing dimmings of 0.011, 0.015, $0.028,0.030$ and 0.034 magnitudes. Again the star is rotated with a period of five hours and observed as 2M1334 was. The rotation period is detected by the cleaned power spectrum (Fig. 22), yet the phased light curve is very nonsinusoidal (Fig. 21c). Note that the power in the rotation period is reduced compared to the previous simulation.

A third simulation is shown in Fig. 23, which is due to a star with eight spots rotating with a period of ten hours. Here the contrast of the individual spots is much smaller, only -0.008 to +0.014 magnitudes. The sampling and noise from 2M1145 (00-02 run) is used and results in a significant variability detection according to the $\chi^{2}$ test, but one close to the variable/non-variable cut-off with $p=0.005$. Despite this low SNR, the rotation period still clearly stands out in the cleaned power spectrum (Fig. 24).

We have carried out many tens of simulations of stars with between one and ten spots with contrasts between -0.1 and +0.1 magnitudes and having random phases, and sampled them using the samping functions of several objects in this paper. We found that provided 

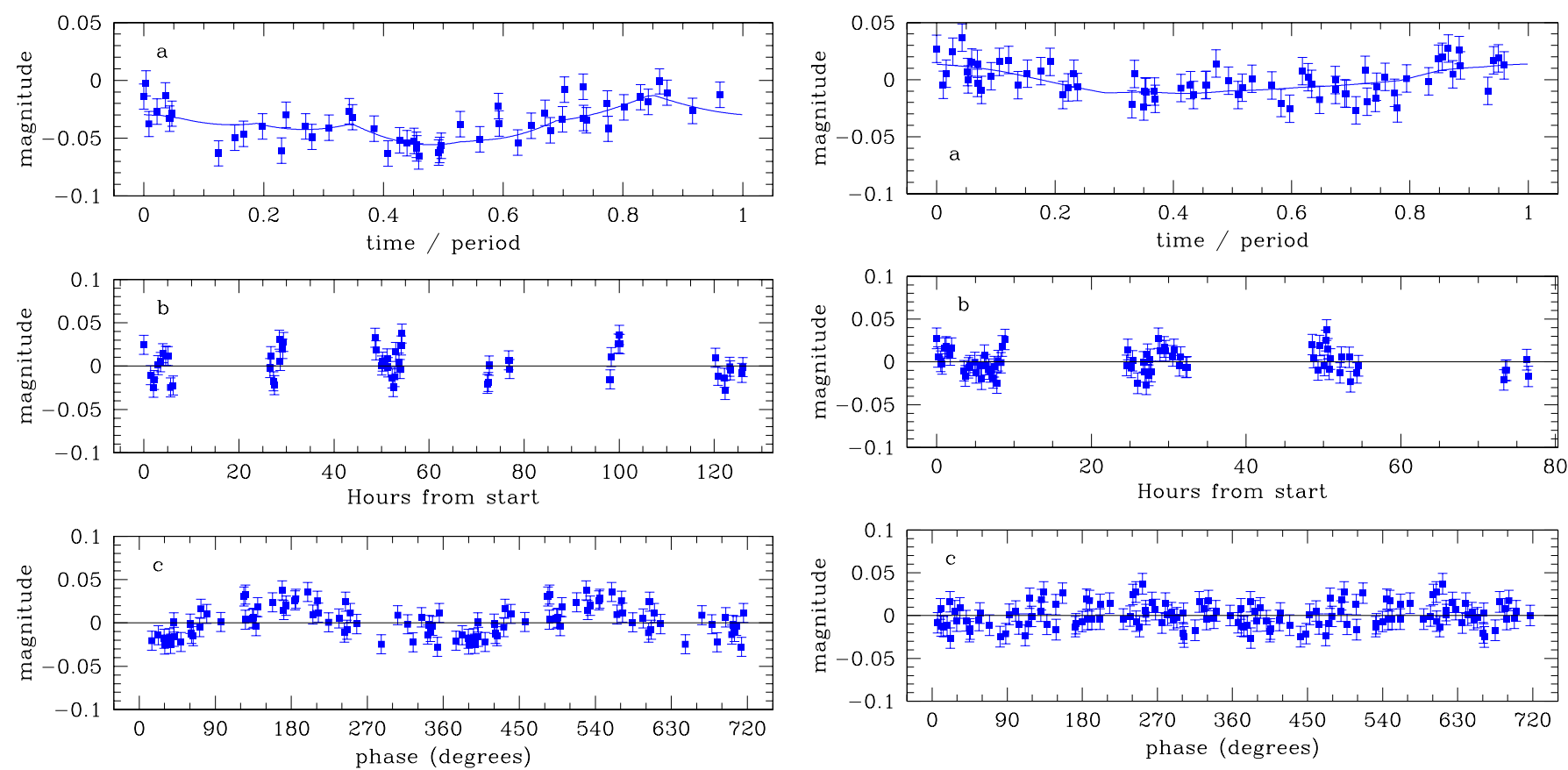

Fig. 21. Same as Fig. 19 except now for five dark spots with random phases. The sampled light curve is again significant $(p<1 \mathrm{e}-9)$, and the cleaned power spectrum (Fig. 22) detects the rotation period at $5.03 \pm 0.10$ hours

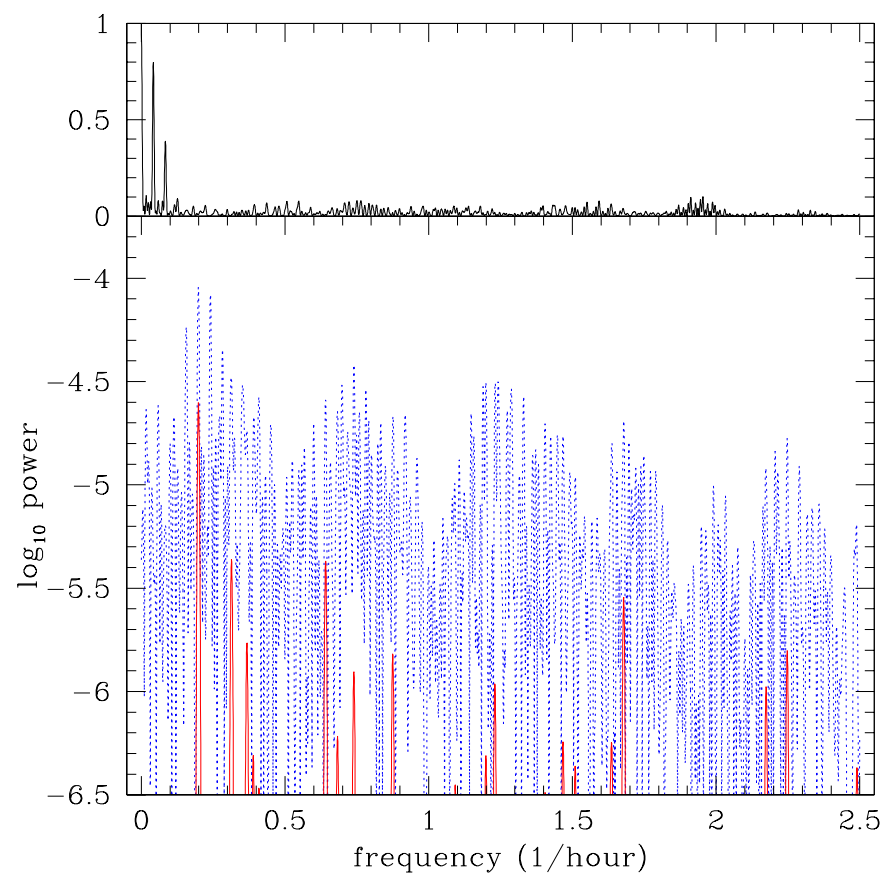

Fig. 22. Power spectrum for the simulated light curve shown in Fig. 21b. The noise level is $\log _{10}(P)=-6.2$. See caption to Fig. 2

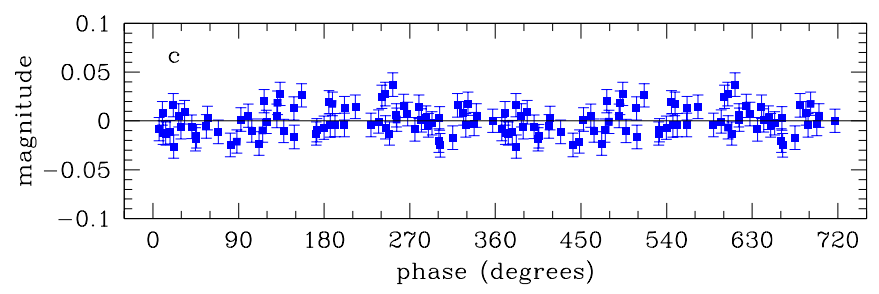

Fig. 23. Same as Fig. 19 except now for eight dark and bright spots with random phases and sampling and noise from 2M1145 (00-02 run). This gives a significant detection, although not overwhelming $(p=0.005)$, yet the cleaned power spectrum (Fig. 24) still detects the rotation period of 10 hours

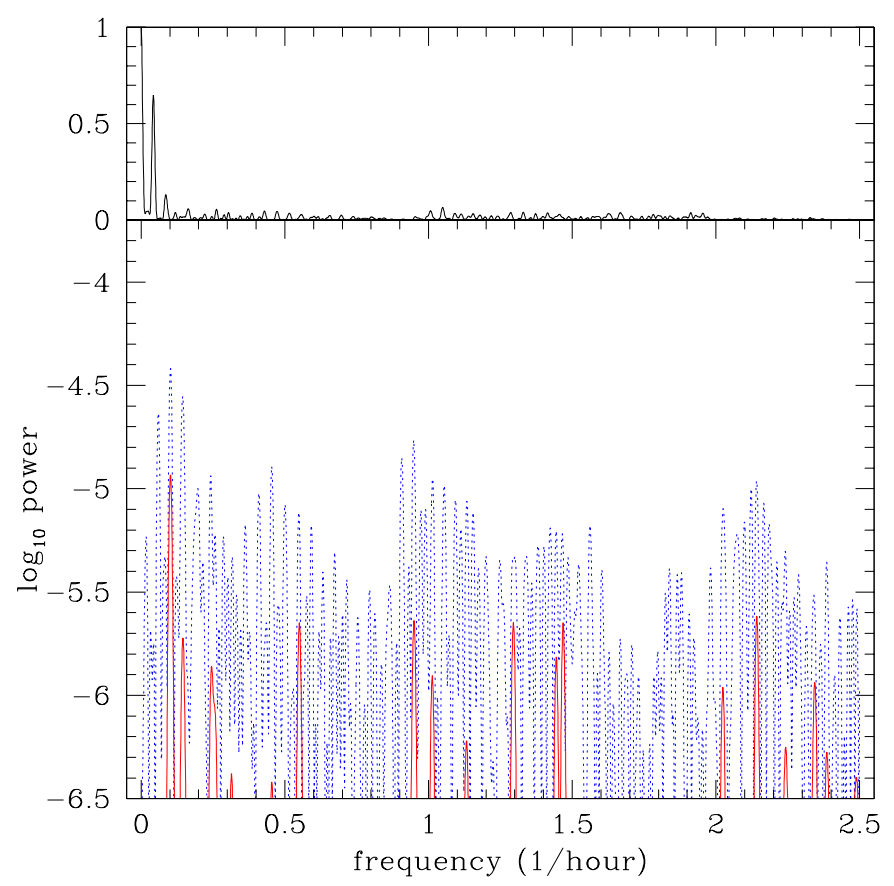

Fig. 24. Power spectrum for the simulated light curve shown in Fig. 23b. The noise level is $\log _{10}(P)=-6.1$. See caption to Fig. 2

\subsection{Evidence for the evolution of surface features}

the light curve showed significant variation (according to our $\chi^{2}$ criterion) then the rotation period was always significant ( $>10$ times the noise level), and in all but one case was the largest peak.

In the light of these simulations, we see that the absence of sinusoidal variation in the light curve phased to a certain period does not rule that out as the rotation period. Thus the phased light curve is not a robust means of identifying 
rotation periods. Moreover, the absence of any significant peaks seems to imply one of two things: either the object is not rotating at a period to which we are sensitive, or the surface features themselves are not stable over the timescale of observations. A third possibility - that the contrast of the spots is too low - is ruled out because we have already made a significant detection of variability according to the $\chi^{2}$ test $^{8}$.

If the duration of observations is less than a rotation period, the light curve will show features rotating on and off the limb of the (unresolved) projected disk: these changes must be represented somehow in the power spectrum, even though they are not due to long-term periodicities of the source. If the surface features are not stable, then the light curve may be even more complex due to the evolution of individual features. In both cases, we may not expect to see any dominant periodicities.

Our maximum time span of observations, $t_{\max }$, is between 30 and 120 hours, so for us to have observed less than a rotation period, all of our objects would have to have maximum $v \sin i$ values of between 1 and $4 \mathrm{kms}^{-1}$ (assuming a radius of $0.1 R_{\odot}$ ). However, this is inconsistent with the results of Basri et al. (2000), who report much higher $v \sin i$ values $\left(10-60 \mathrm{~km} \mathrm{~s}^{-1}\right)$ for all but one of a sample of 17 late $M$ and $L$ dwarfs in the field which were not selected with any known bias for rapid rotation ${ }^{9}$. Thus our objects probably have rotation periods of order 1 to 10 hours, to which we were certainly sensitive. Thus the fact that we have several objects which show no dominant periodicities is significant, as it appears not to be explainable by rotational modulation of stable spots. The logical conclusion from the above arguments is that some of our objects have surface features which evolve over the duration of our observations. This applies in particular to 2M0345, 2M0913, 2M1145 and Calar 3. For 2M1145 we possibly have more direct evidence of this, as the two light curves from one year apart show no common periods, despite the fact that simulations demonstrate we would have detected any likely period due to stable spots in both runs. We can imagine that if the features are themselves changing in brightness then these could dominate the power spectrum and mask the rotation period. The rotation period could possibly then be determined through more measurements over many rotation periods, as the noise level in the power spectrum would then decrease, whereas the power in the rotation period would stay constant.

\footnotetext{
8 Another option is that the rotation period is less than our lower time limit, but this would imply an equatorial rotation speed in excess of $240 \mathrm{~km} \mathrm{~s}^{-1}$.

9 The periods and rotation velocities can be made consistent if the modulating features are at a distance of a solar radius from the rotation axis. However, even the youngest, warmest objects in our sample will have a radius of no more than $0.2 R \odot$ (Chabrier \& Baraffe 2000).
}

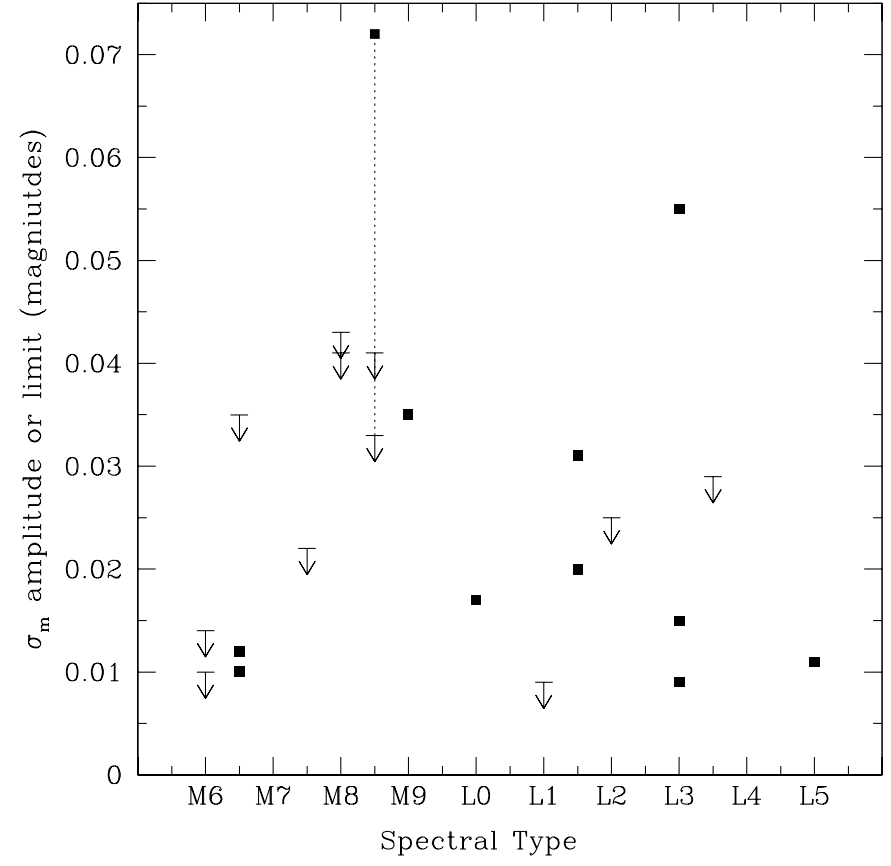

Fig. 25. Relationship between variability amplitudes (squares) or upper limits to variability (arrows) and spectral type. S Ori 45 (M8.5) is plotted as both an amplitude and a limit (connected with a dotted line) depending on whether the first night of data is included or not. The plot using $\overline{\left|m_{\mathrm{d}}\right|}$ rather than $\sigma_{\mathrm{m}}$ as the amplitude measure is very similar

\subsection{Speculation to the physical nature of the surface features}

Variability in stable stars is often attributed to rotational modulation of star spots produced by magnetic activity. In solar-type stars it is believed to be due to the so-called $\alpha \Omega$ dynamo. This mechanism no longer operates in low mass stars and brown dwarfs, but as these objects are fully convective, a turbulent dynamo could come into operation (see Chabrier \& Baraffe 2000 and references therein). M stars often show significant chromospheric activity, as measured by $\log \left(L_{\mathrm{H} \alpha} / L_{\mathrm{bol}}\right)$. Recent work suggests that this value drops from around -3.8 for M7 down to below -6 for L1 and later-type objects (Gizis et al. 2000). Basri (2000) observed a similar decline and Kirkpatrick et al. (2000) detected no $\mathrm{H} \alpha$ emission $(E W>2.0 \AA)$ for types later than L4.5. This suggests that magneticallyinduced surface features may be present on the surfaces of some of the objects in our sample, but that the contrast of the spots may decline beyond M7. This is interesting when we compare it with the relationship between the amplitude of variability and spectral type, shown in Fig. 25. We see that a larger fraction of the objects beyond M9 show variability: 7 of 10 equal to or later than M9, compared to 2 of 9 earlier than M9 (ignoring the ambiguous detection/non-detection in S Ori 45). This is not simply due a higher detection limit for the earlier type objects, as these have an average amplitude/detection limit $\left(\sigma_{\mathrm{m}}\right)$ of $0.025 \mathrm{mag}$, compared to $0.023 \mathrm{mag}$ for the later type objects. If the variability were due to magnetic spots, we 
might expect variability to be less common among the less active later-type objects, not more common as seen here. This trend may be an age effect, as all of our objects of type M9 and earlier are cluster members with ages less than $120 \mathrm{Myr}$. We see no significant relationships between variability amplitude (or limit) and $\mathrm{H} \alpha$ equivalent width.

Another candidate for producing variability is photospheric dust clouds. It is now well established from detailed modelling of optical and infrared spectra that late M and L dwarfs have sufficiently cool atmospheres for solid particles to form (e.g. Jones \& Tsuji 1997; Burrows \& Sharp 1999; Lodders 1999; Chabrier et al. 2000). Whether this dust stays in suspension in the atmosphere or gravitationally settles on a short timescale is still an open question. Basri et al. (2000) conclude that there must be relatively little dust opacity on account of the very strong alkali lines in the optical spectra of $\mathrm{L}$ dwarfs. However, this leaves open the possibility that dust is present deeper in the photosphere where it would affect the infrared spectrum. Models which include dust opacity give better fits to the near infrared spectra of late $\mathrm{M}$ and early $\mathrm{L}$ dwarfs than those which do not (Chabrier et al. 2000). (However, none of the present models predict accurate near infrared colours for late L dwarfs, so it appears that the distribution of dust in the atmospheres of ultra cool dwarfs is more complex than currently appreciated.) Dust may coalesce into large-scale opaque (dark) clouds, and the evolution (formation, growth and dissipation) of such clouds over a few rotation periods could account for our observed variability. These would have to be relatively large clouds, because many small clouds evolving independently would have an insignificant net effect on the light curve. We have seen that ultra cool dwarfs are rapid rotators, and this (as well as possibly differential rotation) is a likely driving mechanism for cloud evolution. These objects are fully convective, so we can imagine a situation in which dust particles are convectively cycled up and down in the photosphere. Dynamical processes such as turbulent diffusion may well be important for modelling dust and its formation into clouds, yet such processes are not taken into account in current atmospheric models. Comparison with weather patterns seen in solar-system atmospheres must be done with caution, however, as solar-system planets are significantly cooler. This dust cloud explanation appears to be supported by our observation that variability is more common in later-type (cooler) objects, i.e. those in which more dust can form.

Other options for the variability can be entertained, such as flaring or outbursts, possibly associated with magnetic activity. $\mathrm{H} \alpha$ flaring is not uncommon in these latetype objects. The very young objects in $\sigma$ Orionis may still have circumstellar disks from which they are accreting matter, and variability of the infall (or even eclipsing by the disk) could account for some variability. There is, however, no evidence for disks from the infrared observations (Zapatero Osorio et al. 2000). Another possible explanation is that the variability is due to hotspots from infalling material in an interacting binary, but this is unlikely to be the explanation in all cases.

Given the relatively small amounts of data on any one object, it is difficult to say much about the characteristics of the variability. However, some of the power spectra are not much different from random data. If we simulate random light curves from a measured light curve by reassigning flux measurements to epochs, we find that the cleaned power spectra often have peaks more than several times the noise. While some peaks reported in Sect. 5 could well be due to noise, not all peaks can be due to noise when we have a significant $\chi^{2}$ detections. There are several random processes intrinsic to the star which could produce the observed light curves, such as the independent evolution of many surface features. Chaotic processes can also give the appearance of a random process when observed in certain parameter spaces.

\section{Summary}

We have presented light curves for 21 late $\mathrm{M}$ and $\mathrm{L}$ dwarfs to probe variability on timescales between a fraction of an hour to over 100 hours. 11 objects showed evidence for variability at the $99 \%$ confidence level according to a $\chi^{2}$ test, with amplitudes between 0.009 and 0.055 magnitudes (rms). Of these objects, four (2M1145, 2M1334, SDSS 0539, S Ori 31) showed strong evidence for variability (confidence greater than $99.99 \%$ ). It has been shown how a careful data reduction and analysis of the errors ensures the reliability of this test. The ten nondetections have upper limits on their rms amplitudes of between 0.009 and 0.043 magnitudes.

A power spectral analysis was performed on all variable objects using the CLEAN algorithm. In a few cases (2M1146, 2M1334, SDSS 0539, S Ori 31) there were significant periodicities (at $5.1 \pm 0.1,2.68 \pm 0.13,13.3 \pm 1.2$ and $7.5 \pm 0.6$ hours respectively) which dominated the power spectra. For 2M1334, SDSS 0529 and S Ori 31 these may be the rotation periods. We demonstrated with simulations that the rotation period does not necessarily produce sinusoidal variation in the light curve: thus these periods can only be confirmed or refuted with longer-term monitoring with more complete coverage. The 5.1 hour period for $2 \mathrm{M} 1146$ was shown not to be the rotation period on the basis of an inconsistency with the $v \sin i$ measurement of Basri et al. The remaining seven significantly variable light curves did not show dominant periods, and in at least three cases (2M0345, 2M0913, Calar 3) there are not even any significant periods. Our simulations showed that we would have detected any plausible rotation periods for these objects based on $v \sin i$ measurements. We therefore concluded that the lack of significant periods was due to the evolution of the features on timescales shorter than our observation span, and that these "wash out" the rotation period in the power spectrum. 2M1145 showed no common periodicities in two separate significantly variable light curves obtained on year apart, thus supporting this view. 
We found that variability is more common in objects later than M9: 7 of 9 objects later than M9 are variable, compared to only 2 of 9 earlier. This may be related to the observation of Gizis et al. that chromospheric activity declines significantly from M7 to L1, and perhaps points to the variability in the late-type objects having a non-magnetic origin; photospheric dust clouds were highlighted as a likely cause. Gaining more insight into the nature of the variability observed in this paper will be the next challenge.

Acknowledgements. CBJ is very grateful to Harry Lehto for use of his CLEAN code and information on its application and interpretation. CBJ also thanks Bill Herbst for useful discussions and an independent analysis of one of the light curves, and Pablo Cincotta for use of his phase dispersion minimisation code. The authors thank Barrie Jones for comments on a draft manuscript. This work is based on observations made with the $2.2 \mathrm{~m}$ telescope at the German-Spanish Astronomical Center at Calar Alto in Spain.

\section{References}

Bailer-Jones, C. A. L., \& Mundt, R. 1999, A\&A, 348, 800, Paper I

Basri, G. 2000, ASP Conf. Ser., in press

Basri, G., Mohanty, S., Allard, F., et al. 2000, ApJ, 538, 363

Burrows, A., \& Sharp, C. M. 1999, ApJ, 512, 843

Béjar, V. J. S., Zapatero-Osorio, M. R., \& Rebolo, R. 1999, ApJ, 521, 671

Chabrier, G., \& Baraffe, I. 2000, ARA\&A, 33, 337

Chabrier, G., Baraffe, I., Allard, F., \& Hauschildt, P. 2000, ApJ, 542, 464

Cincotta, P. M., Méndez, M., \& Néñez, J. A. 1995, ApJ, 449, 213
Deeming, T. J. 1975, Astrophys. Space Sci., 36, 137

Driscoll, W. G. (ed.) 1978, Handbook of Optics, Optical Society of America/McGraw-Hill, 14

Fan, X., Knapp, G. R., Strauss, M. R., et al. 2000, AJ, 119, 928

Gizis, J. E., Monet, D. G., Reid, I. N., et al. 2000, AJ, 120, 1085

Herbst, W., Bailer-Jones, C. A. L., Mundt, R., et al. 2001, in preparation

Jones, H. R. A., \& Tsuji, T. 1997, ApJ, 480, L39

Kirkpatrick, J. D., Reid, I. N., Liebert, J., et al. 1999, ApJ, 519,802

Kirkpatrick, J. D., Reid, I. N., Liebert, J., et al. 2000, AJ, 120, 447

Koerner, D. W., Kirkpatrick, J. D., McElwain, M. W., \& Bonaventura, N. R. 1999, ApJ, 526, L25

Lodders, K. 1999, ApJ, 519, 793

Martín, E. L., Basri, G., Zapatero-Osorio, M. R., Rebolo, R., \& García López, R. J. 1998, ApJ, 507, L41

Nakajima, T., Tsuji, T., Maihara, T., et al. 2000, PASJ, 52, 87

Rebolo, R., Zapatero-Osorio, M. R., \& Martín, E. L. 1995, Nature, 377, 129

Rebolo, R., Martín, E. L., Basri, G., Marcy, G. W., \& ZapateroOsorio, M. R. 1996, ApJ, 469, L53

Reid, I. N., Kirkpatrick, J. D., Gizis, J. E., et al. 2000, AJ, 119,369

Roberts, D. H., Léhar, J., \& Dreher, J. W. 1987, AJ, 93, 968

Terndrup, D. M., Krishnamurthi, A., Pinsonneault, M. H., \& Stauffer, J. R. 1999, ApJ, 118, 1814

Tinney, C. G., \& Tolley, A. J. 1999, MNRAS, 304, 119

Young, A. T., Genet, R. M., Boyd, L. J., et al. 1991, PASP, 103,221

Zapatero-Osorio, M. R., Rebolo, R., Martín, E. L., et al. 1999, A\&AS, 134, 537

Zapatero-Osorio, M. R., Béjar, V. J. S., Martín, E. L., et al. 2000, Science, 290, 103 